\title{
A Novel Conflict Measurement in Decision Making and Its Application in Fault Diagnosis
}

\author{
Fuyuan Xiao, Member, IEEE, Zehong Cao, Member, IEEE, and Alireza Jolfaei, Senior Member, IEEE
}

\begin{abstract}
Dempster-Shafer evidence (DSE) theory, which allows combining pieces of evidence from different data sources to derive a degree of belief function that is a type of fuzzy measure, is a general framework for reasoning with uncertainty. In this framework, how to optimally manage the conflicts of multiple pieces of evidence in DSE remains an open issue to support decision making. The existing conflict measurement approaches can achieve acceptable outcomes but do not fully consider the optimization at the decision-making level using the novel measurement of conflicts. In this paper, we proposed a novel evidential correlation coefficient (ECC) for belief functions by measuring the conflict between two pieces of evidence in decision making. Then, we investigated the properties of our proposed evidential correlation and conflict coefficients, which are all proven to satisfy the desirable properties for conflict measurement, including nonnegativity, symmetry, boundedness, extreme consistency, and insensitivity to refinement. We also presented several examples and comparisons to demonstrate the superiority of our proposed ECC method. Finally, we applied the proposed ECC in a decision-making application of motor rotor fault diagnosis, which verified the practicability and effectiveness of our proposed novel measurement.
\end{abstract}

Index Terms-Dempster-Shafer evidence theory, Conflict management, Evidential correlation coefficient, Belief function, Fuzzy measure, Basic belief assignments, Decision making, Fault diagnosis.

\section{INTRODUCTION}

Uncertainty is an inherent component in data science and big data, especially in a fuzzy environment [1-3]. How to handle and measure the uncertainty to support decisions in various applications [4-6], ranging from medicine to engineering has attracted considerable attention in recent decades [7, 8]. Several novel fuzzy techniques and systems have been presented for reasoning with and managing uncertainty, including the extended intuitionistic fuzzy sets [9], rough sets [10], $\mathrm{Z}$ numbers [11], evidence theory $[12,13]$, evidential reasoning [14], D numbers [15], $\mathrm{R}$ sets and numbers [16, 17], and other hybrid methods [18]. These theories are applied broadly in various fields, such as image classification [19], medical diagnosis [20, 21], information fusion [22], and decision making $[23,24]$. In these fuzziness-related approaches, one of the most useful tools to handle uncertainty is Dempster-Shafer evidence (DSE) theory [25, 26], which has posed several attractive advantages: 1) quantitatively modeling uncertainty

Corresponding authors: Fuyuan Xiao and Zehong Cao.

F. Xiao is with the School of Computer and Information Science, Southwest University, Chongqing 400715, China (Email: xiaofuyuan@swu.edu.cn).

Z. Cao is with the Discipline of ICT, University of Tasmania, Hobart 7001, Australia (Email: zehong.cao@utas.edu.au).

A. Jolfaei is with the Department of Computing, Macquarie University, Sydney 2109, Australia by means of a basic probability assignment (BBA) [27]; 2) the belief function is a type of fuzzy measure that provides partial information in terms of the appropriate fuzzy measure in relation to an uncertain variable [28, 29]; 3) Dempster's combination rule (DCR) satisfies the commutative and associative laws $[30,31]$; 4) the results generated by the DCR have the characteristic of fault-tolerance and relieve the uncertainty level by the DCR $[32,33]$. Consequently, DSE theory can be of benefit for supporting decision making [34] and has been extensively investigated in extracting the information quality of BBA [35] and evidence reliability evaluation [36, 37].

According to previous studies of evidence theory, considering optimal management of conflicts may improve the accuracy performance at the decision-making level in data science applications [38-40]. Therefore, how to measure the conflict of multiple pieces of evidence has attracted considerable research attention in recent years [41-43], and many related definitions have been presented [44] which can be used for fuzzy system-based industrial application areas. Although the outcomes of current conflict management methods are acceptable in DSE theory, we assume there still remains room for improving decision-making performance at the decision level in terms of the measure and management of conflicts.

Therefore, in this study, we explored a novel conflict measurement in decision making. Here, we proposed a new evidential correlation coefficient (ECC), inspired by Jiang's method [45], to measure the correlation between BBAs in DSE theory, which could be proved, analyzed, and applied in the decision making of data science applications. Specifically, we proposed a new evidential conflict coefficient based on ECC to measure the conflict degree between BBAs. Then, we analyzed and proved that the newly defined evidential conflict coefficient has the desirable properties for conflict measurement, including nonnegativity, symmetry, boundedness, extreme consistency, and insensitivity to refinement. Furthermore, we compared the proposed evidential conflict coefficient with well-known methods and demonstrated a motor rotor fault diagnosis application devised based on the ECC.

The rest of this paper is organized as follows. The preliminaries of evidence theory and some existing conflict measures are briefly introduced in Section III and Section IV, respectively. The new evidential correlation and conflict coefficients are proposed in Section V, and their properties are analyzed and proved. Section VI compares various conflict measures to demonstrate the superiority of the proposed method. In Section VII, a fault diagnosis algorithm is devised based on the new correlation coefficient measure; then, the algorithm is 
applied to solve a motor rotor fault diagnosis problem. Finally, Section VIII concludes this work.

\section{RELATED WORKS}

As we know, the traditional Dempster's conflict coefficient $K$ [25] combines the mass allocated to the empty set, accounting for the conflict among focal elements, but it ignores the global consistency between different pieces of evidence.

To overcome this limitation, George and Pal [46], Jousselme et al. [47] and Cheng and Xiao [48] considered the conflict measure from the nonintersecting parts between different pieces of evidence. Another group of researchers quantified the measure of conflict from an alternative perspective. For instance, Liu [49] designed a two-dimensional conflict model by combining Dempster's conflict coefficient and pignistic probability distance. Daniel [50] considered the plausibility conflict of evidence. Lefevre and Elouedi [51] studied measured conflict by means of the distance between pieces of evidence and the mass of an empty set. Furthermore, some novel strategies, such as divergence measures, have also been leveraged to measure evidential consistency [52-54]. For example, Ma and An [52] quantified the divergence grade of evidence by fuzzy nearness and a correlation coefficient. Xiao [53] measured the divergence of evidence by means of Jensen-Shannon divergence. In addition, some researchers investigated conflict measurement from the perspective of correlation coefficients $[45,55,56]$. For instance, Song et al. [55] defined a correlation coefficient [57] as the cosine of the angle between two vectors of pieces of evidence. Pan and Deng [56] developed a correlation coefficient [58] on the basis of Deng entropy [59]. Jiang [45] discussed the conflict measure by taking into account the nonintersection and the difference among focal elements [60].

In this paper, inspired by Jiang's method [45], we propose a novel conflict measurement in decision making and apply it in fault diagnosis, which can improve decision-making performance at the decision level.

\section{PReliminaries}

Many methods handling uncertainty problems have been presented in recent years [61-63]. As a useful uncertainty reasoning tool, DSE theory $[25,26]$ has been widely applied in various areas, such as decision making [64], classification [65, 66], reasoning [67, 68], and industrial alarm systems $[69,70]$. The basic concepts and definitions $[25,26]$ of DSE theory are described below.

\section{Definition 1 (Frame of discernment)}

Let $\Omega$ be a set of mutually exclusive and collective nonempty events defined by $[25,26]$

$$
\Omega=\left\{F_{1}, F_{2}, \ldots, F_{i}, \ldots, F_{n}\right\},
$$

where $\Omega$ is a frame of discernment (FOD).

The power set of $\Omega$ is denoted as $2^{\Omega}$.

$$
\begin{array}{r}
2^{\Omega}=\left\{\emptyset,\left\{F_{1}\right\},\left\{F_{2}\right\}, \ldots,\left\{F_{n}\right\},\left\{F_{1}, F_{2}\right\}, \ldots,\left\{F_{1},\right.\right. \\
\left.\left.F_{2}, \ldots, F_{i}\right\}, \ldots, \Omega\right\},
\end{array}
$$

where $\emptyset$ represents an empty set.
If $A_{i} \in 2^{\Omega}, A_{i}$ is called a hypothesis.

Definition 2 (Mass function)

A mass function $m$ in FOD $\Omega$ can be described as a mapping from $2^{\Omega}$ to $[0,1][25,26]$ :

$$
m: \quad 2^{\Omega} \rightarrow[0,1],
$$

satisfying:

$$
m(\emptyset)=0, \text { and } \sum_{A_{i} \subseteq \Omega} m\left(A_{i}\right)=1 .
$$

In DSE theory, $m$ is also called a BBA. For $A_{i} \subseteq \Omega$, if $m\left(A_{i}\right)$ is greater than zero, $A_{i}$ is called a focal element. Since a BBA can effectively express the uncertainty, various BBA operations have been devised, including negation $[71,72]$ and an entropy function [73].

\section{Definition 3 (Belief function)}

The belief function of $A_{i} \subseteq \Omega$, denoted as $\operatorname{Bel}\left(A_{i}\right)$, is defined as [25, 26]

$$
\operatorname{Bel}\left(A_{i}\right)=\sum_{A_{h} \subseteq A_{i}} m\left(A_{h}\right) .
$$

Definition 4 (Plausibility function)

The plausibility function of $A_{i} \subseteq \Omega$, denoted as $\operatorname{Pl}\left(A_{i}\right)$, is defined as [25, 26]

$$
P l\left(A_{i}\right)=\sum_{A_{h} \cap A_{i} \neq \emptyset} m\left(A_{h}\right) .
$$

$\operatorname{Bel}\left(A_{i}\right)$ and $\operatorname{Pl}\left(A_{i}\right)$ represent the lower and upper bound functions of $A_{i}$, respectively. An interval-valued belief structure can be used for an uncertainty measure $[74,75]$.

\section{Definition 5 (Dempster's combination rule)}

Let $m_{1}$ and $m_{2}$ be two independent BBAs in FOD $\Omega$. Dempster's combination rule (DCR), represented in the form $m=m_{1} \oplus m_{2}$, is defined as $[25,26]$

$m\left(A_{i}\right)= \begin{cases}\frac{1}{1-K} \sum_{A_{h} \cap A_{k}=A_{i}} m_{1}\left(A_{h}\right) m_{2}\left(A_{k}\right), & A_{i} \neq \emptyset, \\ 0, & A_{i}=\emptyset,\end{cases}$

with

$$
K=\sum_{A_{h} \cap A_{k}=\emptyset} m_{1}\left(A_{h}\right) m_{2}\left(A_{k}\right),
$$

where $A_{h}, A_{k} \subseteq \Omega$ and $K$ is the coefficient of conflict between BBAs $m_{1}$ and $m_{2}$.

\section{EXISTING CONFLICT MEASURES}

In this section, some existing conflict measures for belief functions are briefly introduced.

Let $m_{1}$ and $m_{2}$ be two BBAs with hypotheses $A_{i}$ and $A_{j}$, respectively, on the same FOD $\Omega=\left\{F_{1}, \ldots, F_{i}, \ldots, F_{n}\right\}$.

Definition 6 Jousselme et al.'s distance [47]:

$$
d_{J G B}\left(m_{1}, m_{2}\right)=\sqrt{\frac{1}{2}\left(\vec{m}_{1}-\vec{m}_{2}\right)^{T} \underline{\underline{D}}\left(\vec{m}_{1}-\vec{m}_{2}\right)},
$$


where $\vec{m}_{1}$ and $\vec{m}_{2}$ are the BBAs in vector notation and $\underline{D}$ is $a 2^{n} \times 2^{n}$ matrix with elements

$$
D\left(A_{i}, A_{j}\right)=\frac{\left|A_{i} \cap A_{j}\right|}{\left|A_{i} \cup A_{j}\right|},
$$

in which $|\cdot|$ represents the cardinality function.

Definition 7 Lefèvre and Elouedi's adapted conflict [51]:

$$
k_{L E}\left(m_{1}, m_{2}\right)=d_{J G B}\left(m_{1}, m_{2}\right) \cdot m_{\cap}(\emptyset),
$$

where $m_{\cap}(\emptyset)$ is equal to $K$ in $E q$. (8) and $d_{J G B}$ is Eq. (9).

Definition 8 Song et al.'s correlation coefficient [55]:

$$
c_{S W}\left(m_{1}, m_{2}\right)=\frac{<m_{1}^{\prime}, m_{2}^{\prime}>}{\left\|m_{1}^{\prime}\right\| \cdot\left\|m_{2}^{\prime}\right\|},
$$

in which $m^{\prime}$ is defined as

$$
\left\{\begin{array}{l}
m_{1}^{\prime}=m_{1} D \\
m_{2}^{\prime}=m_{2} D
\end{array}\right.
$$

where $D$ is defined in Eq. (10).

Song et al.'s conflict coefficient:

$$
k_{S W}\left(m_{1}, m_{2}\right)=1-c_{S W}\left(m_{1}, m_{2}\right) .
$$

Definition 9 Jiang's correlation coefficient [45]:

$$
c_{J}\left(m_{1}, m_{2}\right)=\frac{c\left(m_{1}, m_{2}\right)}{\sqrt{c\left(m_{1}, m_{1}\right) c\left(m_{2}, m_{2}\right)}},
$$

where $c\left(m_{1}, m_{2}\right)$ is defined as

$$
c\left(m_{1}, m_{2}\right)=\sum_{i=1}^{2^{n}} \sum_{j=1}^{2^{n}} m_{1}\left(A_{i}\right) m_{2}\left(A_{j}\right) \frac{\left|A_{i} \cap A_{j}\right|}{\left|A_{i} \cup A_{j}\right|} .
$$

Jiang's conflict coefficient:

$$
k_{J}\left(m_{1}, m_{2}\right)=1-c_{J}\left(m_{1}, m_{2}\right) .
$$

Definition 10 Cheng and Xiao's distance [48]:

$$
d_{C X}\left(m_{1}, m_{2}\right)=\sqrt{\frac{1}{2}\left(\vec{m}_{1}-\vec{m}_{2}\right)^{T} \underline{\underline{D_{\alpha}}}\left(\vec{m}_{1}-\vec{m}_{2}\right)},
$$

where $\underline{\underline{D_{\alpha}}}$ is a $2^{n} \times 2^{n}$ matrix with elements

$$
D_{\alpha}\left(A_{i}, A_{j}\right)=\frac{\left|A_{i} \cap A_{j}\right|}{\left|A_{i}\right|} \frac{\left|A_{i} \cap A_{j}\right|}{\left|A_{j}\right|} .
$$

\section{THE NEW EVIDENTIAL CORRELATION AND CONFLICT COEFFICIENTS}

For developing an effective conflict measurement, firstly our proposed a new method aims to satisfy the properties of a conflict measurement. Secondly, we consider determining how conflict identification between BBAs for improving performance. Thirdly, for two arbitrary BBAs $m_{1}$ and $m_{2}$, we explore the conflict from the view of $m_{1}$ to $m_{2}$, as well as the conflict from the view of $m_{2}$ to $m_{1}$. Based on the above context, inspired by Jiang's work [45], we design the evidential correlation and conflict coefficients, and specifically address an ECC for measuring the correlation between BBAs. We then analyze and prove the properties of ECC. Furthermore, we define an evidential conflict coefficient and discuss desirable properties for conflict management.

Definition 11 (ECC measure between BBAs)

Let $m_{1}$ and $m_{2}$ be two BBAs on $\Omega=\left\{F_{1}, \ldots, F_{i}, \ldots, F_{n}\right\}$, where $A_{i}$ and $A_{j}$ are hypotheses of BBAs. The ECC between $B B A s m_{1}$ and $m_{2}$, denoted as $E C C\left(m_{1}, m_{2}\right)$, is defined as

$$
\begin{aligned}
\operatorname{ECC}\left(m_{1}, m_{2}\right) & =\cos \Theta\left(\vec{m}_{1}, \vec{m}_{2}\right) \cdot \cos \Theta\left(\vec{m}_{2}, \vec{m}_{1}\right) \\
& =\frac{\left\langle\vec{m}_{1}, \vec{m}_{2}\right\rangle}{\left\|\vec{m}_{1}\right\|\left\|\vec{m}_{2}\right\|} \cdot \frac{\left\langle\vec{m}_{2}, \vec{m}_{1}\right\rangle}{\left\|\vec{m}_{2}\right\|\left\|\vec{m}_{1}\right\|},
\end{aligned}
$$

In Eq. (20), $\cos \Theta$ is a cosine angle function between $\vec{m}_{1}$ and $\vec{m}_{2}$ :

$$
\cos \Theta\left(\vec{m}_{1}, \vec{m}_{2}\right)=\frac{\left\langle\vec{m}_{1}, \vec{m}_{2}\right\rangle}{\left\|\vec{m}_{1}\right\|\left\|\vec{m}_{2}\right\|},
$$

which has a mathematical formula similar to Eq. (12) [55]; $\left\langle\vec{m}_{1}, \vec{m}_{2}\right\rangle$ is the inner product of $\vec{m}_{1}$ and $\vec{m}_{2}$ [45]:

$$
\left\langle\vec{m}_{1}, \vec{m}_{2}\right\rangle=\vec{m}_{1} \cdot \vec{m}_{2}=\sum_{i=1}^{2^{n}} \sum_{j=1}^{2^{n}} m_{1}\left(A_{i}\right) m_{2}\left(A_{j}\right) \frac{\left|A_{i} \cap A_{j}\right|}{\left|A_{i} \cup A_{j}\right|} ;
$$

and $\|\vec{m}\|$ is the norm of $\vec{m}$ :

$$
\|\vec{m}\|=[\langle\vec{m}, \vec{m}\rangle]^{\frac{1}{2}}=\left[\sum_{i=1}^{2^{n}} \sum_{j=1}^{2^{n}} m\left(A_{i}\right) m\left(A_{j}\right) \frac{\left|A_{i} \cap A_{j}\right|}{\left|A_{i} \cup A_{j}\right|}\right]^{\frac{1}{2}} .
$$

Since $\left\langle\vec{m}_{2}, \vec{m}_{1}\right\rangle$ is equal to:

$$
\left\langle\vec{m}_{2}, \vec{m}_{1}\right\rangle=\sum_{j=1}^{2^{n}} \sum_{i=1}^{2^{n}} m_{2}\left(A_{j}\right) m_{1}\left(A_{i}\right) \frac{\left|A_{j} \cap A_{i}\right|}{\left|A_{j} \cup A_{i}\right|},
$$

it can be seen that $\left\langle\vec{m}_{1}, \vec{m}_{2}\right\rangle=\left\langle\vec{m}_{2}, \vec{m}_{1}\right\rangle$.

Hence, Eq. (20) can be expressed in another form:

$$
\operatorname{ECC}\left(m_{1}, m_{2}\right)=\left[\cos \Theta\left(\vec{m}_{1}, \vec{m}_{2}\right)\right]^{2}=\left[\frac{\left\langle\vec{m}_{1}, \vec{m}_{2}\right\rangle}{\left\|\vec{m}_{1}\right\|\left\|\vec{m}_{2}\right\|}\right]^{2} .
$$

Theorem 1 The ECC has the properties of nonnegativity, nondegeneracy, symmetry, and boundedness [45].

Property 1 Let $m_{1}$ and $m_{2}$ be two arbitrary BBAs:

P1.1 Nonnegativity: $\operatorname{ECC}\left(m_{1}, m_{2}\right) \geq 0$.

P1.2 Nondegeneracy: $\operatorname{ECC}\left(m_{1}, m_{2}\right)=1$ if and only if $m_{1}=m_{2}$.

P1.3 Symmetry: $\operatorname{ECC}\left(m_{1}, m_{2}\right)=E C C\left(m_{2}, m_{1}\right)$.

P1.4 Boundedness: $0 \leq E C C\left(m_{1}, m_{2}\right) \leq 1$.

Proof (P1.1) Consider two arbitrary BBAs $m_{a}$ and $m_{b}$ in $F O D \Omega$; we have

$$
\operatorname{ECC}\left(m_{a}, m_{b}\right)=\left[\frac{\left\langle\vec{m}_{a}, \vec{m}_{b}\right\rangle}{\left\|\vec{m}_{a}\right\|\left\|\vec{m}_{b}\right\|}\right]^{2} .
$$

Clearly, $\operatorname{ECC}\left(m_{a}, m_{b}\right) \geq 0$ can be conducted, which proves the property of nonnegativity of the ECC.

(P1.2) Consider two arbitrary BBAs $m_{a}=m_{b}$ in FOD $\Omega$ with the hypotheses of $A_{i}$ and $A_{j}$; we have

$$
\operatorname{ECC}\left(m_{a}, m_{b}\right)=\frac{\left\langle\vec{m}_{a}, \vec{m}_{a}\right\rangle}{\left\|\vec{m}_{a}\right\|^{2}}=\frac{\left\langle\vec{m}_{b}, \vec{m}_{b}\right\rangle}{\left\|\vec{m}_{b}\right\|^{2}}=1 .
$$


Conversely, consider $\operatorname{ECC}\left(m_{a}, m_{b}\right)=1$; we have

$$
\left[\frac{\left\langle\vec{m}_{a}, \vec{m}_{b}\right\rangle}{\left\|\vec{m}_{a}\right\|\left\|\vec{m}_{b}\right\|}\right]^{2}=1
$$

Then, we obtain

$$
\begin{array}{r}
{\left[\sum_{i=1}^{2^{n}} \sum_{j=1}^{2^{n}} m_{a}\left(A_{i}\right) m_{b}\left(A_{j}\right) \frac{\left|A_{i} \cap A_{j}\right|}{\left|A_{i} \cup A_{j}\right|}\right]^{2}=} \\
\sum_{i=1}^{2^{n}} \sum_{j=1}^{2^{n}} m_{a}\left(A_{i}\right) m_{a}\left(A_{j}\right) \frac{\left|A_{i} \cap A_{j}\right|}{\left|A_{i} \cup A_{j}\right|} . \\
\sum_{i=1}^{2^{n}} \sum_{j=1}^{2^{n}} m_{b}\left(A_{i}\right) m_{b}\left(A_{j}\right) \frac{\left|A_{i} \cap A_{j}\right|}{\left|A_{i} \cup A_{j}\right|} .
\end{array}
$$

This equation is satisfied only for $1 \leq i, j \leq 2^{n}$ :

$$
m_{a}\left(A_{i}\right)=m_{b}\left(A_{i}\right) \quad \text { and } \quad m_{a}\left(A_{j}\right)=m_{b}\left(A_{j}\right),
$$

such that

$$
m_{a}=m_{b} .
$$

Hence, $\operatorname{ECC}\left(m_{a}, m_{b}\right)=1 \Longleftrightarrow m_{a}=m_{b}$, which proves the property of nondegeneracy of the ECC.

(P1.3) Consider two arbitrary BBAs $m_{a}$ and $m_{b}$ in FOD $\Omega$ with the hypotheses of $A_{i}$ and $A_{j}$.

For $\left\langle\vec{m}_{a}, \vec{m}_{b}\right\rangle$, we have

$$
\left\langle\vec{m}_{a}, \vec{m}_{b}\right\rangle=\sum_{i=1}^{2^{n}} \sum_{j=1}^{2^{n}} m_{a}\left(A_{i}\right) m_{b}\left(A_{j}\right) \frac{\left|A_{i} \cap A_{j}\right|}{\left|A_{i} \cup A_{j}\right|} .
$$

Furthermore, for $\left\langle\vec{m}_{b}, \vec{m}_{a}\right\rangle$, we have

$$
\left\langle\vec{m}_{b}, \vec{m}_{a}\right\rangle=\sum_{i=1}^{2^{n}} \sum_{j=1}^{2^{n}} m_{b}\left(A_{j}\right) m_{a}\left(A_{i}\right) \frac{\left|A_{j} \cap A_{i}\right|}{\left|A_{j} \cup A_{i}\right|} .
$$

From Eqs. (26) and (27), it is clear that

$$
\left\langle\vec{m}_{a}, \vec{m}_{b}\right\rangle=\left\langle\vec{m}_{b}, \vec{m}_{a}\right\rangle .
$$

Since

$$
\operatorname{ECC}\left(m_{a}, m_{b}\right)=\left[\frac{\left\langle\vec{m}_{a}, \vec{m}_{b}\right\rangle}{\left\|\vec{m}_{a}\right\|\left\|\vec{m}_{b}\right\|}\right]^{2}
$$

and

$$
\operatorname{ECC}\left(m_{b}, m_{a}\right)=\left[\frac{\left\langle\vec{m}_{b}, \vec{m}_{a}\right\rangle}{\left\|\vec{m}_{b}\right\|\left\|\vec{m}_{a}\right\|}\right]^{2},
$$

it is easy to conclude that

$$
\operatorname{ECC}\left(m_{a}, m_{b}\right)=\operatorname{ECC}\left(m_{b}, m_{a}\right),
$$

which proves the property of symmetry of the ECC.

(P1.4) Consider two arbitrary BBAs $m_{a}$ and $m_{b}$ in FOD $\Omega$.

Since $D$ is a Hermitian positive-definite matrix [45], for a $2^{n} \times 2^{n}$ lower triangular matrix $G$, we have:

$$
D=G^{T} G .
$$

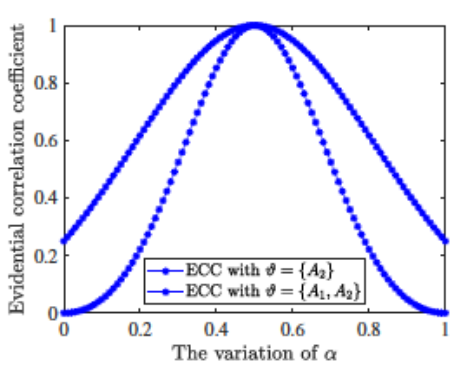

Fig. 1. The evidential correlation coefficient measures in Example 1.

Thus, from [45], we have

$$
\begin{aligned}
& \left\langle\vec{m}_{a}, \vec{m}_{a}\right\rangle=u^{T} D u=u^{T} G^{T} G u ; \\
& \left\langle\vec{m}_{a}, \vec{m}_{b}\right\rangle=u^{T} D v=u^{T} G^{T} G v ; \\
& \left\langle\vec{m}_{b}, \vec{m}_{b}\right\rangle=v^{T} D v=v^{T} G^{T} G v .
\end{aligned}
$$

Thus,

$$
E C C\left(m_{a}, m_{b}\right)=\frac{\left(u^{T} G^{T} G v\right)^{2}}{\left(u^{T} G^{T} G u\right)\left(v^{T} G^{T} G v\right)} .
$$

Because $u^{T} G^{T} G u=(G u)^{T}(G u)=\|G u\|^{2}$, by applying the triangle inequality on the vector 2-norm [45], we obtain

$$
\begin{array}{r}
\|G(u+v)\|^{2} \leq(\|G u\|+\|G v\|)^{2} \Longrightarrow \\
(u+v)^{T} G^{T} G(u+v) \leq\left(\sqrt{u^{T} G^{T} G u}+\sqrt{v^{T} G^{T} G v}\right)^{2} \Longrightarrow \\
u^{T} G^{T} G u+u^{T} G^{T} G v+v^{T} G^{T} G u+v^{T} G^{T} G v \leq u^{T} G^{T} G u+ \\
v^{T} G^{T} G v+2 \sqrt{u^{T} G^{T} G u v^{T} G^{T} G v} \Longrightarrow \\
u^{T} G^{T} G v \leq \sqrt{u^{T} G^{T} G u v^{T} G^{T} G v} \Longrightarrow \\
\frac{\left(u^{T} G^{T} G v\right)^{2}}{u^{T} G^{T} G u v^{T} G^{T} G v} \leq 1 .
\end{array}
$$

Hence, as proved in (P1.1) that $\operatorname{ECC}\left(m_{a}, m_{b}\right) \geq 0$, we obtain

$$
0 \leq \operatorname{ECC}\left(m_{a}, m_{b}\right) \leq 1,
$$

which proves the property of boundedness of the ECC.

Remark 1 Note that the larger $\operatorname{ECC}\left(m_{1}, m_{2}\right)$ is, the greater the correlation coefficient between the BBAs. Therefore, if $\operatorname{ECC}\left(m_{1}, m_{2}\right)=1$, then $m_{1}$ and $m_{2}$ are completely correlated; if $\operatorname{ECC}\left(m_{1}, m_{2}\right)=0$, then $m_{1}$ and $m_{2}$ are completely uncorrelated.

Next, an example is presented to illustrate the properties of $\operatorname{ECC}\left(m_{1}, m_{2}\right)$.

Example 1 Assume there are two BBAs, $m_{1}$ and $m_{2}$, in $\Omega$ :

$$
\begin{array}{ll}
m_{1}: & m_{1}\left(\left\{A_{1}\right\}\right)=\alpha, m_{1}(\vartheta)=1-\alpha ; \\
m_{2}: & m_{2}\left(\left\{A_{1}\right\}\right)=1-\alpha, m_{2}(\vartheta)=\alpha .
\end{array}
$$

In Example 1, $m_{1}$ and $m_{2}$ change according to $\alpha$ and the subset of $\vartheta$. Here, $\alpha$ is set within [0,1], and the subset $\vartheta$ is set as $\left\{A_{2}\right\}$ and $\left\{A_{1}, A_{2}\right\}$. The corresponding correlation coefficient measures are shown in Fig. 1 .

When $\alpha=0.5$, we have $m_{1}\left(\left\{A_{1}\right\}\right)=m_{2}\left(\left\{A_{1}\right\}\right)=0.5$ and $m_{1}(\vartheta)=m_{2}(\vartheta)=0.5$. Regardless of whether the subset 


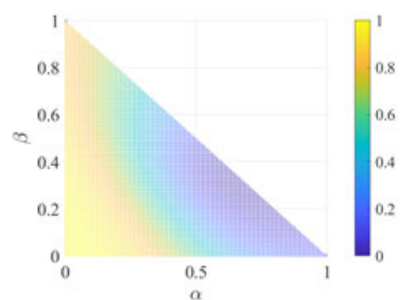

(a) The variation in $\alpha$ and $\beta$.

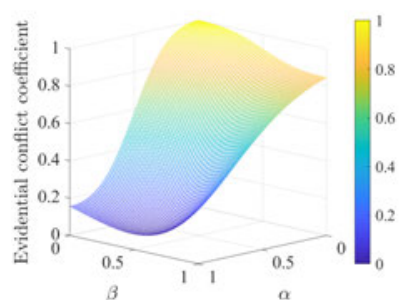

(b) The $k_{E C C}$ under variation in $\alpha$ and $\beta$.

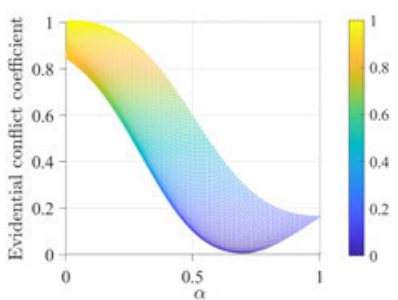

(c) The $k_{E C C}$ under variation in $\alpha$.

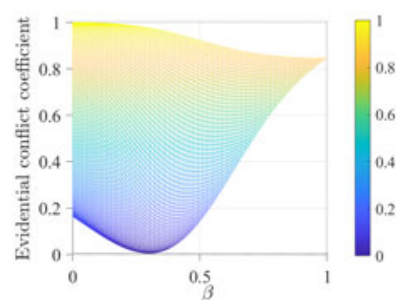

(d) The $k_{E C C}$ under variation in $\beta$.

Fig. 2. The evidential conflict coefficient in Example 2.

$\vartheta$ is $\left\{A_{2}\right\}$ or $\left\{A_{1}, A_{2}\right\}$, the correlation coefficient measures $\operatorname{ECC}\left(m_{1}, m_{2}\right)$ have the maximum value of 1 since $m_{1}$ and $m_{2}$ are the same, that is, completely correlated. Hence, the nondegeneracy property of the ECC is verified. If and only if $m_{1}=m_{2}$, the $\operatorname{ECC}\left(m_{1}, m_{2}\right)$ has the largest correlation coefficient value of 1 .

Furthermore, when $\alpha=0$, and $\vartheta=\left\{A_{2}\right\}$, we have $m_{1}\left(\left\{A_{1}\right\}\right)=m_{2}\left(\left\{A_{2}\right\}\right)=0$ and $m_{1}\left(\left\{A_{2}\right\}\right)=m_{2}\left(\left\{A_{1}\right\}\right)=$ 1 ; when $\alpha=1$, and $\vartheta=\left\{A_{2}\right\}$, we have $m_{1}\left(\left\{A_{1}\right\}\right)=$ $m_{2}\left(\left\{A_{2}\right\}\right)=1$ and $m_{1}\left(\left\{A_{2}\right\}\right)=m_{2}\left(\left\{A_{1}\right\}\right)=0$. Under these two cases, the correlation coefficient measures $\operatorname{ECC}\left(m_{1}, m_{2}\right)$ have the minimum value of 0 since $m_{1}$ and $m_{2}$ are completely uncorrelated.

Moreover, when $\alpha=0$ and $\vartheta=\left\{A_{1}, A_{2}\right\}$, we have $m_{1}\left(\left\{A_{1}\right\}\right)=m_{2}\left(\left\{A_{1}, A_{2}\right\}\right)=0$ and $m_{1}\left(\left\{A_{1}, A_{2}\right\}\right)=$ $m_{2}\left(\left\{A_{1}\right\}\right)=1$; when $\alpha=1$, and $\vartheta=\left\{A_{1}, A_{2}\right\}$, we have $m_{1}\left(\left\{A_{1}\right\}\right)=m_{2}\left(\left\{A_{1}, A_{2}\right\}\right)=1$ and $m_{1}\left(\left\{A_{1}, A_{2}\right\}\right)=$ $m_{2}\left(\left\{A_{1}\right\}\right)=0$. Under these two cases, the $\operatorname{ECC}\left(m_{1}, m_{2}\right)$ measures have a minimal value of 0.25 . This result is reasonable since when $\vartheta=\left\{A_{1}, A_{2}\right\}$, the subsets between $m_{1}\left(\left\{A_{1}\right\}\right)$ and $m_{2}\left(\left\{A_{1}, A_{2}\right\}\right)$ and between $m_{2}\left(\left\{A_{1}\right\}\right)$ and $m_{1}\left(\left\{A_{1}, A_{2}\right\}\right)$ have an intersection of $\left\{A_{1}\right\}$. Hence, $\operatorname{ECC}\left(m_{1}, m_{2}\right)$ is equal to 0.25 rather than zero.

As $\alpha$ increases from 0 to 0.5 , regardless of the subset $\vartheta=$ $\left\{A_{2}\right\}$ or $\vartheta=\left\{A_{1}, A_{2}\right\}, \operatorname{ECC}\left(m_{1}, m_{2}\right)$ gradually increases. This satisfies the expected result since $m_{1}$ and $m_{2}$ become similar as $\alpha$ increases from 0 to 0.5 . On the other hand, as $\alpha$ increases from 0.5 to 1 , regardless of the subset $\vartheta=\left\{A_{2}\right\}$ or $\vartheta=\left\{A_{1}, A_{2}\right\}, E C C\left(m_{1}, m_{2}\right)$ gradually decreases. This also satisfies the intuitive result, since $m_{1}$ and $m_{2}$ become dissimilar when $\alpha$ increases from 0.5 to 1 .

Additionally, in this example, the boundedness property of the ECC, in which $E C C\left(m_{1}, m_{2}\right)$ is greater than or equal to 0 and less than or equal to 1 , is verified. Furthermore, the results shown in Fig. 1 reveal the symmetry property of the ECC.

Based on Definition 11, the evidential conflict coefficient between BBAs is defined as follows.

Definition 12 (The evidential conflict coefficient between $B B A s)$

The evidential conflict coefficient between BBAs $m_{1}$ and $m_{2}$, denoted as $k_{E C C}\left(m_{1}, m_{2}\right)$, is defined as

$k_{E C C}\left(m_{1}, m_{2}\right)=1-E C C\left(m_{1}, m_{2}\right)=1-\left[\frac{\left\langle\vec{m}_{1}, \vec{m}_{2}\right\rangle}{\left\|\vec{m}_{1}\right\|\left\|\vec{m}_{2}\right\|}\right]_{(28)}^{2}$.

Theorem 2 The $k_{E C C}$ has desirable properties for conflict measurement [45], including nonnegativity, symmetry, boundedness, extreme consistency, and insensitivity to refinement.

Property 2 Let $m_{1}$ and $m_{2}$ be two arbitrary BBAs:

P2.1 Nonnegativity: $k_{E C C}\left(m_{1}, m_{2}\right) \geq 0$.

P2.2 Symmetry: $k_{E C C}\left(m_{1}, m_{2}\right)=k_{E C C}\left(m_{2}, m_{1}\right)$.

P2.3 Boundedness: $0 \leq k_{E C C}\left(m_{1}, m_{2}\right) \leq 1$.

P2.4 Extreme consistency: 1) $k_{E C C}\left(m_{1}, m_{2}\right)=1$ iff for the focal elements $A_{i}$ and $A_{j}$ of $m_{1}$ and $m_{2}$, respectively, $\left(\cup A_{i}\right) \cap$ $\left(\cup A_{j}\right)=\emptyset$;2) $k_{E C C}\left(m_{1}, m_{2}\right)=0$ iff $m_{1}$ is completely equal to $m_{2}$.

P2.5 Insensitivity to refinement: for $m_{1}$ and $m_{2}$ refined from FODs $\Omega$ to $\Omega^{\prime}, k_{E C C}\left(m_{1}^{\Omega}, m_{2}^{\Omega}\right)=k_{E C C}\left(m_{1}^{\Omega^{\prime}}, m_{2}^{\Omega^{\prime}}\right)$.

Proof The proofs of (P2.1)-(P2.5) are trivial.

Remark 2 Note that the larger $k_{E C C}\left(m_{1}, m_{2}\right)$ is, the greater the conflict coefficient between the BBAs. If $k_{E C C}\left(m_{1}, m_{2}\right)=1$, then $m_{1}$ and $m_{2}$ are in complete conflict; if $k_{E C C}\left(m_{1}, m_{2}\right)=0$, then $m_{1}$ and $m_{2}$ are in no conflict.

Next, an example is presented to illustrate the nonnegativity and boundedness properties of $k_{E C C}$.

Example 2 Assume there are two BBAs $m_{1}$ and $m_{2}$ in $\Omega$ :

$m_{1}: m_{1}\left(\left\{A_{1}\right\}\right)=\alpha, m_{1}\left(\left\{A_{2}\right\}\right)=\beta, m_{1}\left(\left\{A_{3}\right\}\right)=1-\alpha-\beta ;$ $m_{2}: m_{2}\left(\left\{A_{1}\right\}\right)=0.7, m_{2}\left(\left\{A_{2}\right\}\right)=0.3$.

In Example 2, $m_{1}$ changes according to $\alpha$ and $\beta$, which are set within $[0,1]$ and satisfy $\alpha+\beta \leq 1$, as shown in Fig. 2(a). Then, as $\alpha$ and $\beta$ vary, the corresponding correlation coefficient measures are shown in Figs. 2(b), 2(c) and 2(d).

Fig. 2 verifies the nonnegativity and boundedness properties of $k_{E C C}$, where $k_{E C C} \geq 0$ and $0 \leq k_{E C C} \leq 1$.

As shown in Fig. 2(b), when $\alpha=0.7$ and $\beta=$ 0.3 , we have $m_{1}\left(\left\{A_{1}\right\}\right)=m_{2}\left(\left\{A_{1}\right\}\right)=0.7$ and $m_{1}\left(\left\{A_{2}\right\}\right)=m_{2}\left(\left\{A_{2}\right\}\right)=0.3$. The correlation conflict measure $k_{E C C}\left(m_{1}, m_{2}\right)$ has the smallest value of 0 since $m_{1}$ and $m_{2}$ are exactly the same, that is, completely not in conflict. On the other hand, when $\alpha=\beta=0$, we have $m_{1}\left(\left\{A_{1}\right\}\right)=m_{2}\left(\left\{A_{2}\right\}\right)=0$ and $m_{1}\left(\left\{A_{3}\right\}\right)=1$. In this 


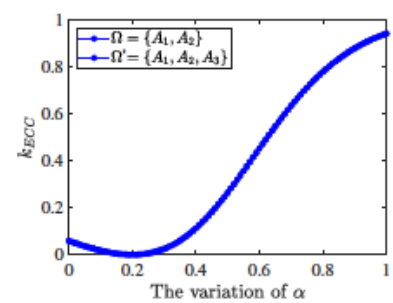

(a) $\gamma=0.2$.

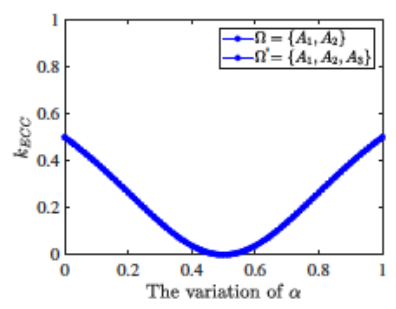

(b) $\gamma=0.5$.

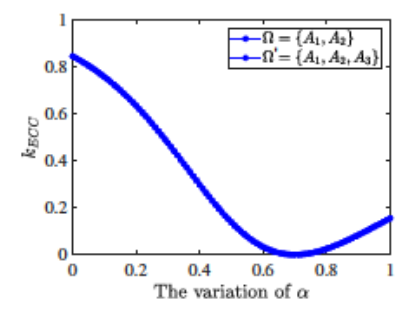

(c) $\gamma=0.7$.

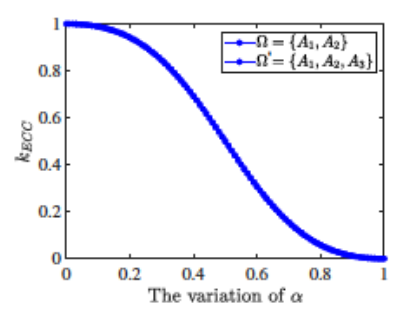

(d) $\gamma=1$.

Fig. 3. The evidential conflict coefficient in Example 3.

case, the correlation conflict measure $k_{E C C}\left(m_{1}, m_{2}\right)$ has the largest value of 1 since $m_{1}$ and $m_{2}$ are completely in conflict. Hence, the extreme consistency property of $k_{E C C}$ is verified in this example.

Furthermore, Fig. 2(c) shows the variation in $k_{E C C}$ as $\alpha$ increases from 0 to 1 . Clearly, when $\alpha$ increases from 0 to 0.7 , since $m_{1}$ gradually becomes closer to $m_{2}$, the conflict coefficient $k_{E C C}$ decreases. As $\alpha$ increases from 0.7 to 1 , because $m_{1}$ tends to become dissimilar to $m_{2}$, the conflict coefficient $k_{E C C}$ increases.

Moreover, Fig. 2(d) shows the variation in $k_{E C C}$ as $\beta$ increases from 0 to 1 . Similarly, when $\beta$ increases from 0 to 0.3 , since $m_{1}$ becomes closer to $m_{2}$, the conflict coefficient $k_{E C C}$ decreases. As $\beta$ increases from 0.3 to 1 , because $m_{1}$ shifts farther from $m_{2}$, the conflict coefficient $k_{E C C}$ increases.

Next, we present an example to illustrate the symmetry and insensitivity to refinement properties of $k_{E C C}$.

Example 3 Assume there are two BBAs $m_{1}$ and $m_{2}$ in $\Omega=$ $\left\{A_{1}, A_{2}\right\}$ and $\Omega^{\prime}=\left\{A_{1}, A_{2}, A_{3}\right\}$, respectively:

$$
\begin{array}{cc}
m_{1}: & m_{1}\left(\left\{A_{1}\right\}\right)=\alpha, m_{1}\left(\left\{A_{2}\right\}\right)=1-\alpha ; \\
m_{2}: & m_{2}\left(\left\{A_{1}\right\}\right)=\gamma, m_{2}\left(\left\{A_{2}\right\}\right)=1-\gamma .
\end{array}
$$

In Example 3, $m_{1}$ and $m_{2}$ change in accordance with the variation in $\alpha$ and $\gamma$, respectively. $\alpha$ is set within $[0,1]$ for $m_{1}$. For $m_{2}, \gamma$ is set as $0.2,0.5,0.7$ and 1 . Additionally, $m_{1}$ and $m_{2}$ have the same subsets and support values under $\Omega$ and $\Omega^{\prime}$. Then, as $\alpha$ and $\gamma$ vary, the corresponding correlation coefficient measures under $\Omega$ and $\Omega^{\prime}$ are shown in Fig. 3 .

As shown in Figs. 3(a), 3(b), 3(c) and 3(d), $k_{E C C}$ is not impacted by the variation in the FODs from $\Omega$ to $\Omega^{\prime}$. Even under variation in $\alpha$ and $\gamma$, the value of $k_{E C C}$ under the FOD $\Omega$ is always the same as that under the FOD $\Omega^{\prime}$. Moreover, when we change the input $k_{E C C}\left(m_{1}, m_{2}\right)$ to $k_{E C C}\left(m_{2}, m_{1}\right)$, the results are exactly the same. Consequently, the symmetry and insensitivity to refinement properties of $k_{E C C}$ are verified.

\section{COMPARISON WITH EXISTING METHODS}

In this section, several numerical examples are provided to illustrate the characteristics of different conflict measures, namely, the traditional Dempster's $K$ [25], Jousselme et al.'s $d_{J G B}$ [47], Lefèvre and Elouedi's $k_{L E}$ [51], Song et al.'s $k_{S W}$ [55], Jiang's $k_{J}$ [45], Cheng and Xiao's $d_{C X}$ [48] and the proposed $k_{E C C}$. In addition, we assess whether these conflict measures satisfy the abovementioned properties, as well as the degrees of conflict.
Example 4 Assume there are two BBAs $m_{1}$ and $m_{2}$ in $\Omega=$ $\left\{A_{1}, A_{2}, A_{3}, A_{4}\right\}$ :

$$
\begin{array}{ll}
m_{1}: & m_{1}\left(\left\{A_{1}\right\}\right)=0.5, m_{1}\left(\left\{A_{2}\right\}\right)=0.5, \\
& m_{1}\left(\left\{A_{3}\right\}\right)=0.0, m_{1}\left(\left\{A_{4}\right\}\right)=0.0 ; \\
m_{2}: & m_{2}\left(\left\{A_{1}\right\}\right)=0.0, m_{2}\left(\left\{A_{2}\right\}\right)=0.0, \\
& m_{2}\left(\left\{A_{3}\right\}\right)=0.5, m_{2}\left(\left\{A_{4}\right\}\right)=0.5 .
\end{array}
$$

In Example 4, for the focal elements $A_{i}$ and $A_{j}$ of $m_{1}$ and $m_{2}$, respectively, we have $\left(\cup A_{i}\right) \cap\left(\cup A_{j}\right)=\emptyset$. Thus, $m_{1}$ and $m_{2}$ are in complete conflict, so the conflict grade between $m_{1}$ and $m_{2}$ is assumed to be 1 .

TABLE I

THE CONFLICT DEGREES GENERATED BY DIFFERENT METHODS IN EXAMPLE 4.

\begin{tabular}{llllllll}
\hline BBAs & $K$ & $d_{J G B}$ & $k_{L E}$ & $k_{S W}$ & $k_{J}$ & $d_{C X}$ & $k_{E C C}$ \\
\hline$\left(m_{1}, m_{2}\right)$ & 1 & 0.7071 & 0.7071 & 0.6010 & 1 & 0.7071 & 1 \\
\hline
\end{tabular}

The results in Table I indicate that the conflict degrees produced by $K, k_{J}$ and $k_{E C C}$ are 1 , in accordance with the intuitive result. By contrast, $d_{J G B}, k_{L E}$ and $d_{C X}$ generate a conflict value of 0.7071 , and $k_{S W}$ has a conflict degree of 0.601 . Therefore, $d_{J G B}, k_{L E}, k_{S W}$ and $d_{C X}$ do not satisfy the extreme consistency property of conflict measures.

Example 5 Assume there are two BBAs $m_{1}$ and $m_{2}$ in $\Omega=$ $\left\{A_{1}, A_{2}, A_{3}, A_{4}\right\}$ :

$$
\begin{array}{ll}
m_{1}: & m_{1}\left(\left\{A_{1}\right\}\right)=0.25, m_{1}\left(\left\{A_{2}\right\}\right)=0.25, \\
& m_{1}\left(\left\{A_{3}\right\}\right)=0.25, m_{1}\left(\left\{A_{4}\right\}\right)=0.25 ; \\
m_{2}: & m_{2}\left(\left\{A_{1}\right\}\right)=0.25, m_{2}\left(\left\{A_{2}\right\}\right)=0.25, \\
& m_{2}\left(\left\{A_{3}\right\}\right)=0.25, m_{2}\left(\left\{A_{4}\right\}\right)=0.25 .
\end{array}
$$

In Example 5, $m_{1}$ is the same as $m_{2}$, with the same support values for the corresponding subsets. Therefore, $m_{1}$ and $m_{2}$ are completely nonconflicting, so the conflict grade between $m_{1}$ and $m_{2}$ should be zero.

TABLE II

THE CONFLICT DEGREES GENERATED BY DIFFERENT METHODS IN EXAMPLE 5.

\begin{tabular}{llllllll}
\hline BBAs & $K$ & $d_{J G B}$ & $k_{L E}$ & $k_{S W}$ & $k_{J}$ & $d_{C X}$ & $k_{E C C}$ \\
\hline$\left(m_{1}, m_{2}\right)$ & 0.75 & 0 & 0 & 0 & 0 & 0 & 0 \\
\hline
\end{tabular}


Table II shows that the conflict degrees calculated by $d_{J G B}$, $k_{L E}, k_{S W}, k_{J}, d_{C X}$ and $k_{E C C}$ are zero, in agreement with the expected result. By contrast, $K$ generates a conflict value of 0.75 , which does not satisfy the extreme consistency property of conflict measures.

Example 6 Assume there are two BBAs $m_{1}$ and $m_{2}$ in $\Omega=$ $\left\{A_{1}, A_{2}\right\}$ and $\Omega^{\prime}=\left\{A_{1}, A_{2}, A_{3}\right\}$, respectively:

$$
\begin{array}{ll}
m_{1}: & m_{1}\left(\left\{A_{1}\right\}\right)=0.8, m_{1}\left(\left\{A_{2}\right\}\right)=0.2 ; \\
m_{2}: & m_{2}\left(\left\{A_{1}\right\}\right)=0.2, m_{2}\left(\left\{A_{2}\right\}\right)=0.8 .
\end{array}
$$

In Example 6, the different FODs $\Omega=\left\{A_{1}, A_{2}\right\}$ and $\Omega^{\prime}=$ $\left\{A_{1}, A_{2}, A_{3}\right\}$ have the same BBAs $m_{1}$ and $m_{2}$. Intuitively, the conflict grade between $m_{1}$ and $m_{2}$ on $\Omega$ and $\Omega^{\prime}$ should be the same.

TABLE III

THE CONFLICT DEGREES GENERATED BY DIFFERENT METHODS IN EXAMPLE 6.

\begin{tabular}{llllllll}
\hline & $K$ & $d_{J G B}$ & $k_{L E}$ & $k_{S W}$ & $k_{J}$ & $d_{C X}$ & $k_{E C C}$ \\
\hline$\Omega$ & 0.6800 & 0.6000 & 0.4080 & 0.3871 & 0.5294 & 0.6000 & 0.7785 \\
$\Omega^{\prime}$ & 0.6800 & 0.6000 & 0.4080 & 0.3716 & 0.5294 & 0.6000 & 0.7785 \\
\hline
\end{tabular}

As shown in Table III, $K=0.68, d_{J G B}=0.6, k_{L E}=$ $0.408, k_{J}=0.5294, d_{C X}=0.6$ and $k_{E C C}=0.7785$, regardless of FOD $\Omega$ or $\Omega^{\prime}$. This conforms to the expected result. However, $k_{S W}$ generates a conflict value of 0.3871 on FOD $\Omega$ and conflict of 0.3716 on FOD $\Omega^{\prime}$, which does not satisfy the conflict measure property of insensitivity to refinement.

In summary, the above examples demonstrate the disadvantages of the conflict measures derived in related works. $k_{J}$ and the proposed $k_{E C C}$ satisfy the properties of conflict measures, especially the last two properties. By contrast, $K, d_{J G B}, k_{L E}$, $k_{S W}$ and $d_{C X}$ do not satisfy the extreme consistency property, and $k_{S W}$ does not satisfy the property of insensitivity to refinement. To further study the effectiveness of the proposed $k_{E C C}$, we discuss the following examples.

Example 7 Assume there exist two BBAs $m_{1}$ and $m_{2}$ in $\Omega=$ $\left\{A_{1}, A_{2}, \ldots, A_{19}\right\}$ :

$$
\begin{aligned}
m_{1}: & m_{1}(\Omega)=0.1, m_{1}\left(\left\{A_{2}, A_{3}, A_{4}\right\}\right)=0.05, \\
& m_{1}\left(\left\{A_{7}\right\}\right)=0.05, m_{1}\left(\vartheta_{i}\right)=0.8 \\
m_{2}: & m_{2}\left(\left\{A_{1}, A_{2}, A_{3}, A_{4}, A_{5}\right\}\right)=1 .
\end{aligned}
$$

In Example 7, the subset $\vartheta_{i}$ of $m_{1}$ changes from $\left\{A_{1}\right\}$ to $\left\{A_{1}, \ldots, A_{19}\right\}$, as shown in Table IV. Note that $m_{2}$ has one focal element such that $m_{2}\left(\left\{A_{1}, A_{2}, A_{3}, A_{4}, A_{5}\right\}\right)=1$. Then, the conflict measures between BBAs $m_{1}$ and $m_{2}$ are calculated, as shown in Fig. 4 and Table V.

When $i=5$, the support value of subset $\left\{A_{1}, A_{2}, A_{3}, A_{4}, A_{5}\right\}$ of $m_{1}$ is 0.8 , which is closer to that of $m_{2}$ with the subset $\left\{A_{1}, A_{2}, A_{3}, A_{4}, A_{5}\right\}$ than in other cases of $i$. Therefore, the expected conflict grade is assumed to achieve the minimum value. The results in Fig. 4 indicate that when $\vartheta_{i}$ of $m_{1}$ varies from $\left\{A_{1}\right\}$ to $\left\{A_{1}, A_{2}, A_{3}, A_{4}, A_{5}\right\}$, the $d_{J G B}, k_{L E}, k_{J}, d_{C X}$ and $k_{E C C}$ decrease, where $d_{J G B}$,
TABLE IV

THE VARIATION IN $\vartheta_{i}$

\begin{tabular}{ll}
\hline$i$ & $\vartheta_{i}$ \\
\hline 1 & $\left\{A_{1}\right\}$ \\
2 & $\left\{A_{1}, A_{2}\right\}$ \\
3 & $\left\{A_{1}, A_{2}, A_{3}\right\}$ \\
4 & $\left\{A_{1}, A_{2}, A_{3}, A_{4}\right\}$ \\
5 & $\left\{A_{1}, A_{2}, A_{3}, A_{4}, A_{5}\right\}$ \\
6 & $\left\{A_{1}, A_{2}, A_{3}, A_{4}, A_{5}, A_{6}\right\}$ \\
7 & $\left\{A_{1}, A_{2}, A_{3}, A_{4}, A_{5}, A_{6}, A_{7}\right\}$ \\
8 & $\left\{A_{1}, A_{2}, A_{3}, A_{4}, A_{5}, A_{6}, A_{7}, A_{8}\right\}$ \\
9 & $\left\{A_{1}, A_{2}, A_{3}, A_{4}, A_{5}, A_{6}, A_{7}, A_{8}, A_{9}\right\}$ \\
10 & $\left\{A_{1}, A_{2}, A_{3}, A_{4}, A_{5}, A_{6}, A_{7}, A_{8}, A_{9}, A_{10}\right\}$ \\
11 & $\left\{A_{1}, A_{2}, A_{3}, A_{4}, A_{5}, A_{6}, A_{7}, A_{8}, A_{9}, A_{10}, A_{11}\right\}$ \\
12 & $\left\{A_{1}, A_{2}, A_{3}, A_{4}, A_{5}, A_{6}, A_{7}, A_{8}, A_{9}, A_{10}, A_{11}, A_{12}\right\}$ \\
13 & $\left\{A_{1}, A_{2}, A_{3}, A_{4}, A_{5}, A_{6}, A_{7}, A_{8}, A_{9}, A_{10}, A_{11}, A_{12}, A_{13}\right\}$ \\
14 & $\left\{A_{1}, A_{2}, A_{3}, A_{4}, A_{5}, A_{6}, A_{7}, A_{8}, A_{9}, A_{10}, A_{11}, A_{12}, \ldots, A_{14}\right\}$ \\
15 & $\left\{A_{1}, A_{2}, A_{3}, A_{4}, A_{5}, A_{6}, A_{7}, A_{8}, A_{9}, A_{10}, A_{11}, A_{12}, \ldots, A_{15}\right\}$ \\
16 & $\left\{A_{1}, A_{2}, A_{3}, A_{4}, A_{5}, A_{6}, A_{7}, A_{8}, A_{9}, A_{10}, A_{11}, A_{12}, \ldots, A_{16}\right\}$ \\
17 & $\left\{A_{1}, A_{2}, A_{3}, A_{4}, A_{5}, A_{6}, A_{7}, A_{8}, A_{9}, A_{10}, A_{11}, A_{12}, \ldots, A_{17}\right\}$ \\
18 & $\left\{A_{1}, A_{2}, A_{3}, A_{4}, A_{5}, A_{6}, A_{7}, A_{8}, A_{9}, A_{10}, A_{11}, A_{12}, \ldots, A_{18}\right\}$ \\
19 & $\left\{A_{1}, A_{2}, A_{3}, A_{4}, A_{5}, A_{6}, A_{7}, A_{8}, A_{9}, A_{10}, A_{11}, A_{12}, \ldots, A_{19}\right\}$ \\
\hline
\end{tabular}

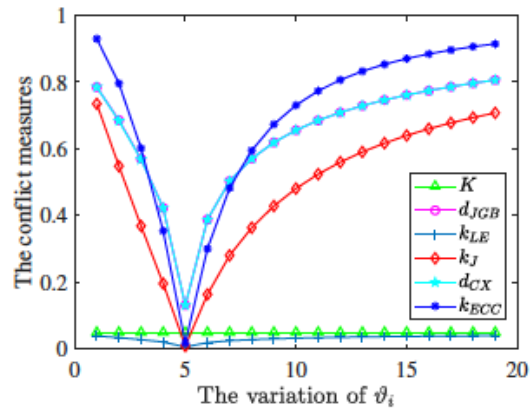

Fig. 4. The different conflict measures in Example 7.

$k_{L E}, k_{J}, d_{C X}$ and $k_{E C C}$ achieve minimum values of 0.1315 , $0.0066,0.0094,0.1315$ and 0.0186 , respectively. As $\vartheta_{i}$ increases from $\left\{A_{1}, A_{2}, A_{3}, A_{4}, A_{5}\right\}$ to $\left\{A_{1}, \ldots, A_{19}\right\}$, the $d_{J G B}, k_{L E}, k_{J}, d_{C X}$ and $k_{E C C}$ conflict measures increase. Nevertheless, $K$ does not perform well in this example: the conflict measure always maintains the same value of 0.05 regardless of the variation in $\vartheta_{i}$.

Example 8 Assume there exist two BBAs $m_{1}$ and $m_{2}$ in $\Omega=$ $\left\{A_{1}, A_{2}, \ldots, A_{19}\right\}$ :

$$
\begin{array}{ll}
m_{1}: & m_{1}\left(\left\{A_{1}\right\}\right)=0.1, m_{1}\left(\vartheta_{i}\right)=0.9 ; \\
m_{2}: & m_{2}\left(\left\{A_{1}\right\}\right)=0.9, m_{2}\left(\vartheta_{i}\right)=0.1 .
\end{array}
$$

In Example 8, the subsets of $m_{1}$ and $m_{2}$ change according to the variation in $\vartheta_{i}$ in Table IV. $m_{1}$ and $m_{2}$ have the same subsets, namely, $\left\{A_{1}\right\}$ and $\vartheta_{i}$, where the subset $\vartheta_{i}$ increases as $i$ varies from 1 to 19 . The conflict degrees between BBAs $m_{1}$ and $m_{2}$ produced by different methods are shown in Fig. 5 .

In the case that $i=1$, we have $m_{1}\left(\left\{A_{1}\right\}\right)=m_{2}\left(\left\{A_{1}\right\}\right)=$ 1. Hence, all the conflict measures have a value of zero. As $i$ increases from 2 to $19, d_{J G B}, k_{J}, d_{C X}$ and $k_{E C C}$ increase, where it is reasonable and intuitive since as $\vartheta_{i}$ varies from 
TABLE V

THE COMPARISON OF CONFLICT DEGREES IN EXAMPLE 7.

\begin{tabular}{lllllll}
\hline$\vartheta_{i}$ & $K$ & $d_{J G B}$ & $k_{L E}$ & $k_{J}$ & $d_{C X}$ & $k_{E C C}$ \\
\hline$\left\{A_{1}\right\}$ & 0.05 & 0.7851 & 0.0393 & 0.7348 & 0.7851 & 0.9297 \\
$\left\{A_{1}, A_{2}\right\}$ & 0.05 & 0.6860 & 0.0343 & 0.5483 & 0.6836 & 0.7959 \\
$\left\{A_{1}, A_{2}, A_{3}\right\}$ & 0.05 & 0.5700 & 0.0285 & 0.3690 & 0.5680 & 0.6018 \\
$\left\{A_{1}, \ldots, A_{4}\right\}$ & 0.05 & 0.4232 & 0.0212 & 0.1964 & 0.4232 & 0.3542 \\
$\left\{A_{1}, \ldots, A_{5}\right\}$ & 0.05 & 0.1315 & 0.0066 & 0.0094 & 0.1315 & 0.0186 \\
$\left\{A_{1}, \ldots, A_{6}\right\}$ & 0.05 & 0.3884 & 0.0194 & 0.1639 & 0.3884 & 0.3009 \\
$\left\{A_{1}, \ldots, A_{7}\right\}$ & 0.05 & 0.5031 & 0.0252 & 0.2808 & 0.5031 & 0.4827 \\
$\left\{A_{1}, \ldots, A_{8}\right\}$ & 0.05 & 0.5709 & 0.0285 & 0.3637 & 0.5709 & 0.5952 \\
$\left\{A_{1}, \ldots, A_{9}\right\}$ & 0.05 & 0.6192 & 0.0310 & 0.4288 & 0.6192 & 0.6737 \\
$\left\{A_{1}, \ldots, A_{10}\right\}$ & 0.05 & 0.6560 & 0.0328 & 0.4811 & 0.6560 & 0.7307 \\
$\left\{A_{1}, \ldots, A_{11}\right\}$ & 0.05 & 0.6852 & 0.0343 & 0.5241 & 0.6852 & 0.7736 \\
$\left\{A_{1}, \ldots, A_{12}\right\}$ & 0.05 & 0.7091 & 0.0355 & 0.5602 & 0.7091 & 0.8065 \\
$\left\{A_{1}, \ldots, A_{13}\right\}$ & 0.05 & 0.7291 & 0.0365 & 0.5908 & 0.7291 & 0.8325 \\
$\left\{A_{1}, \ldots, A_{14}\right\}$ & 0.05 & 0.7463 & 0.0373 & 0.6171 & 0.7463 & 0.8534 \\
$\left\{A_{1}, \ldots, A_{15}\right\}$ & 0.05 & 0.7612 & 0.0381 & 0.6400 & 0.7612 & 0.8704 \\
$\left\{A_{1}, \ldots, A_{16}\right\}$ & 0.05 & 0.7743 & 0.0387 & 0.6600 & 0.7743 & 0.8844 \\
$\left\{A_{1}, \ldots, A_{17}\right\}$ & 0.05 & 0.7861 & 0.0393 & 0.6778 & 0.7861 & 0.8962 \\
$\left\{A_{1}, \ldots, A_{18}\right\}$ & 0.05 & 0.7967 & 0.0398 & 0.6936 & 0.7967 & 0.9061 \\
$\left\{A_{1}, \ldots, A_{19}\right\}$ & 0.05 & 0.8063 & 0.0403 & 0.7078 & 0.8063 & 0.9146 \\
\hline
\end{tabular}

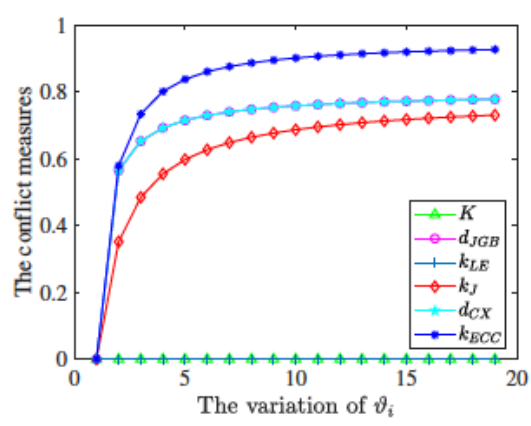

Fig. 5. The different conflict measures in Example 8.

$\left\{A_{1}, A_{2}\right\}$ to $\left\{A_{1}, \ldots, A_{19}\right\}$, the uncertainty increases. However, $K$ and $k_{L E}$ remain at zero, regardless of the variation in $\vartheta_{i}$. On the other hand, as shown by comparison with $d_{J G B}$, $k_{J}$ and $d_{C X}$, the proposed $k_{E C C}$ can better distinguish the conflict between $m_{1}$ and $m_{2}$ with higher values.

TABLE VI

THE VARIATION IN $\delta_{i}$.

\begin{tabular}{ll}
\hline$i$ & $\delta_{i}$ \\
\hline $1-10$ & $\vartheta_{i}$ \\
11 & $\left\{A_{2}, A_{3}, A_{4}, A_{5}, A_{6}, A_{7}, A_{8}, A_{9}, A_{10}\right\}$ \\
12 & $\left\{A_{3}, A_{4}, A_{5}, A_{6}, A_{7}, A_{8}, A_{9}, A_{10}\right\}$ \\
13 & $\left\{A_{4}, A_{5}, A_{6}, A_{7}, A_{8}, A_{9}, A_{10}\right\}$ \\
14 & $\left\{A_{5}, A_{6}, A_{7}, A_{8}, A_{9}, A_{10}\right\}$ \\
15 & $\left\{A_{6}, A_{7}, A_{8}, A_{9}, A_{10}\right\}$ \\
16 & $\left\{A_{7}, A_{8}, A_{9}, A_{10}\right\}$ \\
17 & $\left\{A_{8}, A_{9}, A_{10}\right\}$ \\
18 & $\left\{A_{9}, A_{10}\right\}$ \\
19 & $\left\{A_{10}\right\}$ \\
\hline
\end{tabular}

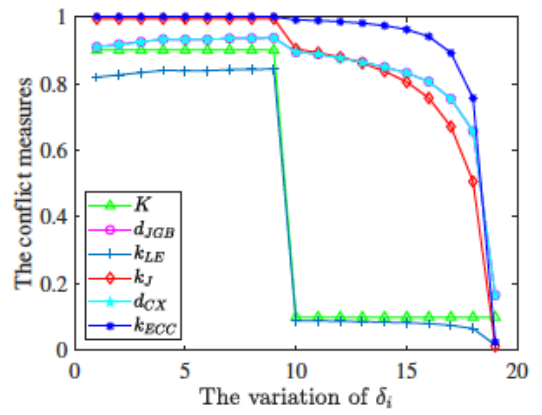

Fig. 6. The different conflict measures in Example 9.

Example 9 Assume there exist two BBAs $m_{1}$ and $m_{2}$ in $\Omega=$ $\left\{A_{1}, A_{2}, \ldots, A_{19}\right\}$ :

$$
\begin{aligned}
m_{1}: & m_{1}(\Omega)=0.1, m_{1}\left(\left\{A_{2}, A_{3}, A_{4}\right\}\right)=0.05, \\
& m_{1}\left(\left\{A_{7}\right\}\right)=0.05, m_{1}\left(\delta_{i}\right)=0.8 ; \\
m_{2}: & m_{2}\left(\left\{A_{10}\right\}\right)=1 .
\end{aligned}
$$

In Example 9, the subset $\delta_{i}$ of $m_{1}$ changes from $\delta_{1}$ to $\delta_{19}$, as shown in Table VI. When $i=1,2, \ldots, 10, \delta_{i}$ is the same as $\vartheta_{i}$. When $i$ increases from 11 to 19 , the subset $\delta_{i}$ of $m_{1}$ is pruned from its first element until it becomes $\left\{A_{10}\right\}$. Note that $m_{2}$ has one focal element such that $m_{2}\left(\left\{A_{10}\right\}\right)=1$. The conflict measures between BBAs $m_{1}$ and $m_{2}$ are calculated and shown in Fig. 6.

As $i$ increases from 1 to 9 , since $m_{1}$ and $m_{2}$ are highly dissimilar, the expected conflict measure is assumed to achieve the maximal value. From Fig. 6, we can see that $K, d_{J G B}$, $k_{L E}, k_{J}, d_{C X}$ and $k_{E C C}$ show the same trend of increasing conflict values. When $i=10, \vartheta_{i}$ first includes $\left\{A_{10}\right\}$. All the conflict measures become smaller than those in the case where $i=1, \ldots, 9$. As $\vartheta_{i}$ increases from 11 to 19 , while the subset decreases from $\left\{A_{2}, A_{3}, A_{4}, A_{5}, A_{6}, A_{7}, A_{8}, A_{9}, A_{10}\right\}$ to $\left\{A_{10}\right\}$, the $d_{J G B}, k_{L E}, k_{J}, d_{C X}$ and $k_{E C C}$ methods have decreasing conflict measures that reach minimal values of $0.1658,0.0166,0.0128,0.1658$ and 0.0255 , respectively. By contrast, $K$ remains unchanged at $K=0.1$, which does not satisfy the intuitive result.

In summary, Examples 4 to 9 clearly show that $k_{E C C}$ is superior to other methods: $k_{E C C}$ not only satisfies all the desired properties but also provides better conflict identification. Consequently, the proposed approach is effective and suitable for measuring conflict between BBAs.

\section{ALgORITHM AND APPLICATION}

Determining how to address decision-making problems has attracted considerable attention in recent years [76, 77]. In this section, a decision-making algorithm for fault diagnosis is devised based on the correlation coefficient measure. Then, a real application of motor rotor fault diagnosis from [45] is used to demonstrate the efficiency of the proposed method.

\section{A. Algorithm}

Problem statement: Let $\left\{F_{1}, \ldots, F_{i}, \ldots, F_{n}\right\}$ be a set of fault types for a kind of machine that establishes an FOD $\Theta$, and let 
$\mathcal{M}=\left\{m_{1}, \ldots, m_{j}, \ldots, m_{k}\right\}$ be $k$ pieces of evidence modeled from the collected data of the sensors. A threshold $\xi$ can be set in advance for making a decision. The goal of the algorithm is to diagnose which type of fault occurs according to the given BBAs $\left\{m_{1}, \ldots, m_{j}, \ldots, m_{k}\right\}$, and threshold $\xi$.

Step 1: A correlation matrix is constructed by leveraging the ECC:

$$
M_{E C C}=\left[\begin{array}{ccc}
E C C\left(m_{1}, m_{1}\right) & \cdots & E C C\left(m_{1}, m_{k}\right) \\
\vdots & \vdots & \vdots \\
E C C\left(m_{k}, m_{1}\right) & \cdots & E C C\left(m_{k}, m_{k}\right)
\end{array}\right] .
$$

Step 2: The support degree of $m_{j}$ is calculated as:

$$
S D\left(m_{j}\right)=\sum_{i=1 \mid i \neq j}^{k} \operatorname{ECC}\left(m_{i}, m_{j}\right) .
$$

Step 3: The credibility degree of $m_{j}$ is calculated as:

$$
C D\left(m_{j}\right)=\frac{S D\left(m_{j}\right)}{\sum_{j=1}^{k} S D\left(m_{j}\right)} .
$$

Step 4: The weighted average evidence (WAE) is obtained as:

$$
W A E(m)=\sum_{j=1}^{k} C D\left(m_{j}\right) \times m_{j} .
$$

Step 5: The WAE is fused $k-1$ times with the DCR:

$$
\operatorname{Fusion}(m)=\left((m \oplus m)_{1} \oplus \cdots \oplus m\right)_{(k-1)} .
$$

Step 6: The $m\left(F_{o}\right)$ with the highest value is selected:

$$
o=\underset{1 \leq i \leq n}{\arg \max }\left\{m\left(F_{i}\right)\right\} .
$$

Step 7: The fault type is determined as follows:

$$
\begin{cases}\text { if } m\left(F_{o}\right) \geq \xi, & F_{o} \text { is the fault type } \\ \text { if } m\left(F_{o}\right)<\xi, & \text { Cannot be determined }\end{cases}
$$

This fault diagnosis based on the ECC is given in Algorithm 1 .

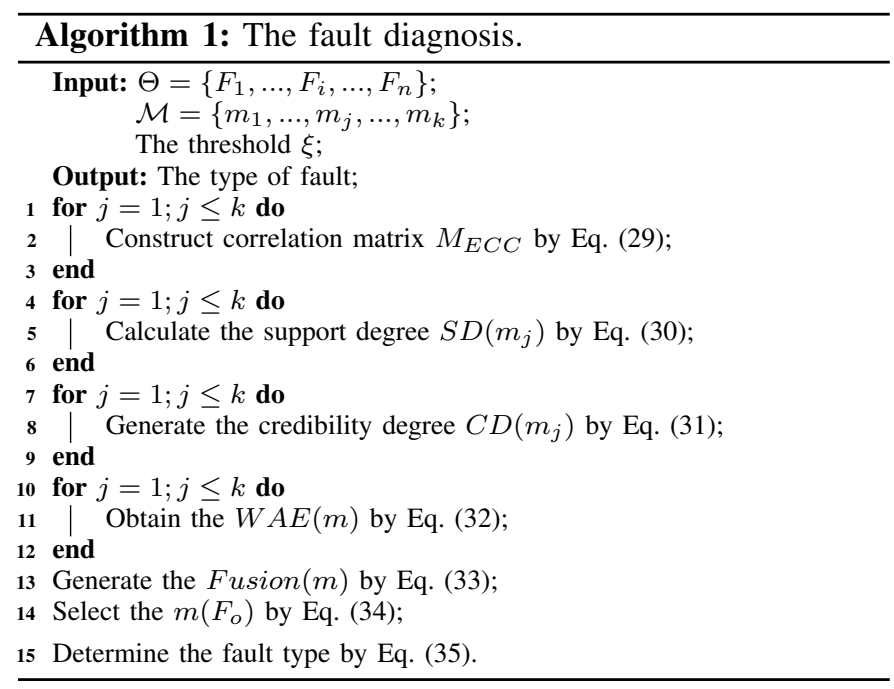

TABLE VII

THE BBAS IN THE APPLICATION OF FAULT DIAGNOSIS.

\begin{tabular}{llllll}
\hline BBAs & $F_{1}$ & $F_{2}$ & $F_{3}$ & $F_{4}$ & $\Theta$ \\
\hline$m_{1}$ & 0.06 & 0.68 & 0.02 & 0.04 & 0.20 \\
$m_{2}$ & 0.02 & 0.00 & 0.79 & 0.05 & 0.14 \\
$m_{3}$ & 0.02 & 0.58 & 0.16 & 0.04 & 0.20 \\
\hline
\end{tabular}

\section{B. Application - fault diagnosis}

In the motor rotor fault diagnosis application [45], three types of sensors are located at different places to collect the acceleration, velocity, and displacement information for a motor rotor. Then, the collected data are modeled as BBAs, as shown in Table VII, where $m_{1}, m_{2}$, and $m_{3}$ represent three pieces of evidence from the sensors. There are four states for a motor rotor, which establishes an FOD $\Theta=\left\{F_{1}, F_{2}, F_{3}, F_{4}\right\}$ : $F_{1}$ represents "normal operation", $F_{2}$ represents "unbalance", $F_{3}$ represents "misalignment", and $F_{4}$ represents "pedestal looseness". In this application, the threshold for making a decision is set to 0.7 based on [45].

A decision is difficult to make based solely on the BBAs $m_{1}, m_{2}$ and $m_{3}$. Specifically, $m_{1}$ has a value of 0.68 , which indicates $F_{2}$ : "unbalance"; $m_{2}$ has a value of 0.79 , which indicates $F_{3}$ : "misalignment"; and $m_{3}$ has a value of 0.58 , which indicates $F_{2}$ : "unbalance". Since $m_{1}\left(F_{2}\right)=0.68$ and $m_{3}\left(F_{2}\right)=0.58$, which are less than the threshold 0.7, a decision cannot be made on the basis of $m_{1}$ and $m_{2}$, whereas according to $m_{3}$, the diagnosis result is $F_{3}$. As a result, conflict exists between $m_{1}, m_{2}$ and $m_{3}$, so an accurate decision is difficult to make under such circumstances. Thus, a conflict management method is necessary to improve the decision level.

Step 1: The correlation matrix $M_{E C C}$ is constructed as:

$$
M_{E C C}=\left[\begin{array}{lll}
1.0000 & 0.0335 & 0.9516 \\
0.0335 & 1.0000 & 0.1517 \\
0.9516 & 0.1517 & 1.0000
\end{array}\right] .
$$

Step 2: The support degree of $m_{j}$ is calculated as:

$$
\begin{aligned}
& S D\left(m_{1}\right)=0.9851 ; S D\left(m_{2}\right)=0.1852 ; \\
& S D\left(m_{3}\right)=1.1033 .
\end{aligned}
$$

Step 3: The credibility degree of $m_{j}$ is calculated as:

$$
\begin{aligned}
& C D\left(m_{1}\right)=0.4333 ; C D\left(m_{2}\right)=0.0815 ; \\
& C D\left(m_{3}\right)=0.4853 .
\end{aligned}
$$

Step 4: The weighted average evidence (WAE) is obtained as:

$$
\begin{aligned}
& m\left(F_{1}\right)=0.0373 ; m\left(F_{2}\right)=0.5761 ; \\
& m\left(F_{3}\right)=0.1507 ; m\left(F_{4}\right)=0.0408 ; \\
& m(\Theta)=0.1951 .
\end{aligned}
$$

Step 5: The WAE is fused 2 times with the DCR:

$$
\begin{aligned}
& m\left(F_{1}\right)=0.0102 ; m\left(F_{2}\right)=0.8964 ; \\
& m\left(F_{3}\right)=0.0674 ; m\left(F_{4}\right)=0.0113 ; \\
& m(\Theta)=0.0148 .
\end{aligned}
$$


TABLE VIII

COMPARISON OF DIFFERENT METHODS IN THE APPLICATION OF FAULT DIAGNOSIS.

\begin{tabular}{lllllll}
\hline Methods & $F_{1}$ & $F_{2}$ & $F_{3}$ & $F_{4}$ & $\Theta$ & Fault type \\
\hline Dempster [25] & 0.0205 & 0.5230 & 0.3933 & 0.0309 & 0.0324 & Cannot be determined \\
Murphy [39] & 0.0112 & 0.6059 & 0.3508 & 0.0153 & 0.0168 & Cannot be determined \\
Deng et al. [40] & 0.0111 & 0.7730 & 0.1856 & 0.0139 & 0.0165 & unbalance \\
Jiang [45] & 0.0108 & 0.8063 & 0.1534 & 0.0134 & 0.0162 & unbalance \\
Proposed method & 0.0102 & 0.8964 & 0.0674 & 0.0113 & 0.0148 & unbalance \\
\hline
\end{tabular}

Step 6: The $m\left(F_{i}\right)$ with the highest value is selected:

$$
o=\underset{1 \leq i \leq n}{\arg \max }\left\{m\left(F_{i}\right)\right\}=2 .
$$

Step 7: Since $m\left(F_{2}\right)=0.8964$, which is greater than the threshold 0.7 , the fault type is $F_{2}$.

\section{Discussion}

To demonstrate the effectiveness of the proposed conflict management method, we compare the proposed method with related works, including Dempster's [25], Murphy's [39], Deng et al. 's [40], and Jiang's [45] methods. The results generated by different conflict management methods are shown in Table VIII. Dempster's and Murphy's methods cannot determine the fault type because their $m\left(F_{2}\right)$ values of 0.5230 and 0.6059 , respectively, are smaller than the threshold of 0.7. On the other hand, the methods of Deng et al. and Jiang and the proposed method can diagnose the fault type of the motor rotor as "unbalance", as they obtain $m\left(F_{2}\right)$ values of $0.7730,0.8063$ and 0.8964 , respectively. Moreover, the proposed method has the highest value of 0.8964 and can thus diagnose the fault type with a higher rate of identification.

\section{CONCLUSIONS}

In this paper, we explored a novel conflict measurement in decision making and its application in fault diagnosis. Here, a new evidential correlation coefficient, called ECC, was proposed for modeling belief functions in evidence theory to support decision making in an uncertain environment. The properties of the ECC were defined and analyzed, and the ECC was confirmed to have the properties of nonnegativity, nondegeneracy, symmetry, and boundedness. Furthermore, on the basis of the ECC, an evidential conflict coefficient was proposed to measure the conflict between two pieces of evidence. The evidential conflict coefficient was proved to have the desired properties for conflict measurement, including nonnegativity, symmetry, boundedness, extreme consistency, and insensitivity to refinement.

We provided several examples to compare our proposed ECC method with the well-known approaches to demonstrate the superiority of this novel conflict measurement. We also applied the ECC in a fault diagnosis application, and the results verified that our proposed conflict measurement is shown to more efficiently handle uncertainty compared with existing approaches. In summary, our proposed conflict measurement provides a promising way to manage conflict from multiple pieces of evidence and improve the performance of decision making, illustrating a good potential alternative to the analysis of big data from multiple sources. In future work, we intend to further study the properties of ECC as well as its application in more complex environments.

\section{CONFLICT OF INTEREST}

The authors state that there are no conflicts of interest.

\section{ACKNOWLEDGMENTS}

The author greatly appreciates the reviewers' suggestions and the editor's encouragement. This research is supported by the Research Project of Education and Teaching Reform of Southwest University (No. 2019JY053), Fundamental Research Funds for the Central Universities (No. XDJK2019C085) and the Chongqing Overseas Scholars Innovation Program (No. cx2018077).

\section{REFERENCES}

[1] H. Fujita, A. Gaeta, V. Loia, and F. Orciuoli, "Hypotheses analysis and assessment in counter-terrorism activities: a method based on OWA and fuzzy probabilistic rough sets," IEEE Transactions on Fuzzy Systems, vol. 28, pp. 831-845, 2019.

[2] M. Ali, F. Feng, T. Mahmood, I. Mahmood, and H. Faizan, "A graphical method for ranking atanassov's intuitionistic fuzzy values using the uncertainty index and entropy," International Journal of Intelligent Systems, vol. 34, no. 10, pp. 2692-2712, 2019.

[3] J. Morente-Molinera, X. Wu, A. Morfeq, R. Al-Hmouz, and E. Herrera-Viedma, "A novel multi-criteria group decision-making method for heterogeneous and dynamic contexts using multi-granular fuzzy linguistic modelling and consensus measures," Information Fusion, vol. 53, pp. 240-250, 2020.

[4] D. Cavaliere, J. A. Morente-Molinera, V. Loia, S. Senatore, and E. Herrera-Viedma, "Collective scenario understanding in a multi-vehicle system by consensus decision making," IEEE Transactions on Fuzzy Systems, p. DOI: 10.1109/TFUZZ.2019.2928787, 2019.

[5] H. Garg and S. Chen, "Multiattribute group decision making based on neutrality aggregation operators of qrung orthopair fuzzy sets," Information Sciences, vol. 517, pp. 427-447, 2020.

[6] D. Pelusi, R. Mascella, L. Tallini, J. Nayak, B. Naik, and A. Abraham, "Neural network and fuzzy system for the tuning of gravitational search algorithm parameters," Expert Systems with Applications, vol. 102, 022018. 
[7] H. Seiti and A. Hafezalkotob, "A new risk-based fuzzy cognitive model and its application to decision-making," Cognitive Computation, vol. 12, no. 1, pp. 309-326, 2020.

[8] F. Xiao, "EFMCDM: Evidential fuzzy multicriteria decision making based on belief entropy," IEEE Transactions on Fuzzy Systems, p. DOI: 10.1109/TFUZZ.2019.2936368, 2019.

[9] H. Garg and K. Kumar, "Linguistic interval-valued atanassov intuitionistic fuzzy sets and their applications to group decision making problems," IEEE Transactions on Fuzzy Systems, vol. 27, no. 12, pp. 2302-2311, 2019.

[10] J. C. Alcantud, F. Feng, and R. Yager, "An N-soft set approach to rough sets," IEEE Transactions on Fuzzy Systems, p. DOI: 10.1109/TFUZZ.2019.2946526, 2019.

[11] W. Jiang, Y. Cao, and X. Deng, "A Novel Znetwork Model Based on Bayesian Network and Znumber," IEEE Transactions on Fuzzy Systems, p. DOI: 10.1109/TFUZZ.2019.2918999, 2019.

[12] F. Xiao, "Generalization of Dempster-Shafer theory: A complex mass function," Applied Intelligence, pp. DOI: 10.1007/s10 489-019-01 617-y, 2019.

[13] — , "Generalized belief function in complex evidence theory," Journal of Intelligent \& Fuzzy Systems, vol. 38, no. 4, pp. 3665-3673, 2020.

[14] Z.-G. Liu, Q. Pan, J. Dezert, and A. Martin, "Combination of classifiers with optimal weight based on evidential reasoning," IEEE Transactions on Fuzzy Systems, vol. 26, no. 3, pp. 1217-1230, 2018.

[15] X. Deng and W. Jiang, "A total uncertainty measure for D numbers based on belief intervals," International Journal of Intelligent Systems, vol. 34, no. 12, pp. 3302-3316, 2019.

[16] H. Seiti, A. Hafezalkotob, and L. Martinez, "R-sets, comprehensive fuzzy sets risk modeling for riskbased information fusion and decision-making," IEEE Transactions on Fuzzy Systems, p. DOI: 10.1109/TFUZZ.2019.2955061, 2019.

[17] H. Seiti, A. Hafezalkotob, and L. Martínez, "R-numbers, a new risk modeling associated with fuzzy numbers and its application to decision making," Information Sciences, vol. 483, pp. 206-231, 2019.

[18] B. Kang, P. Zhang, Z. Gao, G. Chhipi-Shrestha, K. Hewage, and R. Sadiq, "Environmental assessment under uncertainty using Dempster-Shafer theory and Znumbers," Journal of Ambient Intelligence and Humanized Computing, pp. DOI: 10.1007/s12 652-019-01 228y, 2019.

[19] W. Jiang, K. Huang, J. Geng, and X. Deng, "Multi-scale metric learning for few-shot learning," IEEE Transactions on Circuits and Systems for Video Technology, p. DOI: 10.1109/TCSVT.2020.2995754, 2020.

[20] Z. Cao, C.-T. Lin, K.-L. Lai, L.-W. Ko, J.-T. King, K.-K. Liao, J.-L. Fuh, and S.-J. Wang, "Extraction of SSVEPsbased inherent fuzzy entropy using a wearable headband EEG in migraine patients," IEEE Transactions on Fuzzy Systems, p. DOI: 10.1109/TFUZZ.2019.2905823, 2019.

[21] Z. Cao, C.-H. Chuang, J.-K. King, and C.-T. Lin,
"Multi-channel EEG recordings during a sustainedattention driving task," Scientific Data, vol. 6, pp. DOI: 10.1038/s41 597-019-0027-4, 2019.

[22] F. Xiao, "GIQ: A generalized intelligent qualitybased approach for fusing multi-source information," IEEE Transactions on Fuzzy Systems, p. DOI: 10.1109/TFUZZ.2020.2991296, 2020.

[23] H. Seiti, A. Hafezalkotob, and S. E. Najaf, "Developing a novel risk-based MCDM approach based on D numbers and fuzzy information axiom and its applications in preventive maintenance planning," Applied Soft Computing, vol. 82, p. 105559, 2019.

[24] F. Xiao, Z. Zhang, and J. Abawajy, "Workflow scheduling in distributed systems under fuzzy environment," Journal of Intelligent \& Fuzzy Systems, vol. 37, no. 4, pp. 53235333, 2019.

[25] A. P. Dempster, "Upper and lower probabilities induced by a multivalued mapping," Annals of Mathematical Statistics, vol. 38, no. 2, pp. 325-339, 1967.

[26] G. Shafer et al., A mathematical theory of evidence. Princeton University Press Princeton, 1976, vol. 1.

[27] W. Jiang, Z. Zhang, and X. Deng, "A novel failure mode and effects analysis method based on fuzzy evidential reasoning rules," IEEE Access, vol. 7, pp. $113605-$ 113615, 2019.

[28] R. R. Yager, "A class of fuzzy measures generated from a Dempster-Shafer belief structure," International Journal of Intelligent Systems, vol. 14, no. 12, pp. 1239-1247, 1999.

[29] G. Banon, "Distinction between several subsets of fuzzy measures," Fuzzy Sets and Systems, vol. 5, no. 3, pp. 291-305, 1981.

[30] H. Zhang and Y. Deng, "Weighted belief function of sensor data fusion in engine fault diagnosis," Soft Computing, vol. 24, no. 3, pp. 2329-2339, 2020.

[31] L. Fei, Y. Feng, and L. Liu, "Evidence combination using OWA-based soft likelihood functions," International Journal of Intelligent Systems, vol. 34, no. 9, pp. 2269 2290, 2019.

[32] R. R. Yager, "Generalized Dempster-Shafer structures," IEEE Transactions on Fuzzy Systems, vol. 27, no. 3, pp. 428-435, 2019.

[33] M. Zhou, X.-B. Liu, Y.-W. Chen, and J.-B. Yang, "Evidential reasoning rule for MADM with both weights and reliabilities in group decision making," Knowledge-Based Systems, vol. 143, pp. 142-161, 2018.

[34] S. Gao and Y. Deng, "An evidential evaluation of nuclear safeguards," International Journal of Distributed Sensor Networks, vol. 15, no. 12, pp. 1-9, 2019.

[35] X. Cao and Y. Deng, "A new geometric mean FMEA method based on information quality," IEEE Access, vol. 7, no. 1, pp. 95 547-95 554, 2019.

[36] Y. Song, X. Wang, J. Zhu, and L. Lei, "Sensor dynamic reliability evaluation based on evidence theory and intuitionistic fuzzy sets," Applied Intelligence, vol. 48, no. 11, pp. 3950-3962, 2018.

[37] C.-1. Fan, Y. Song, L. Lei, X. Wang, and S. Bai, "Evidence reasoning for temporal uncertain information 
based on relative reliability evaluation," Expert Systems with Applications, vol. 113, pp. 264-276, 2018.

[38] F. Liu, X. Gao, J. Zhao, and Y. Deng, "Generalized belief entropy and its application in identifying conflict evidence," IEEE Access, vol. 7, no. 1, pp. 126625$126633,2019$.

[39] C. K. Murphy, "Combining belief functions when evidence conflicts," Decision support systems, vol. 29, no. 1, pp. 1-9, 2000.

[40] Y. Deng, W. Shi, Z. Zhu, and Q. Liu, "Combining belief functions based on distance of evidence," Decision Support Systems, vol. 38, no. 3, pp. 489-493, 2004.

[41] F. Xiao, "CED: A distance for complex mass functions," IEEE Transactions on Neural Networks and Learning Systems, p. DOI: 10.1109/TNNLS.2020.2984918, 2020.

[42] Y. Song, X. Wang, and H. Zhang, "A distance measure between intuitionistic fuzzy belief functions," Knowledge-Based Systems, vol. 86, pp. 288-298, 2015.

[43] F. Xiao, "A distance measure for intuitionistic fuzzy sets and its application to pattern classification problems," IEEE Transactions on Systems, Man, and Cybernetics: Systems, p. DOI: 10.1109/TSMC.2019.2958635, 2019.

[44] D. Han, J. Dezert, and Y. Yang, "Belief interval-based distance measures in the theory of belief functions," IEEE Transactions on Systems, Man, and Cybernetics: Systems, vol. 48, no. 6, pp. 833-850, 2016.

[45] W. Jiang, "A correlation coefficient for belief functions," International Journal of Approximate Reasoning, vol. 103, pp. 94-106, 2018.

[46] T. George and N. R. Pal, "Quantification of conflict in Dempster-Shafer framework: a new approach," International Journal Of General System, vol. 24, no. 4, pp. 407-423, 1996.

[47] A.-L. Jousselme, D. Grenier, and É. Bossé, "A new distance between two bodies of evidence," Information Fusion, vol. 2, no. 2, pp. 91-101, 2001.

[48] C. Cheng and F. Xiao, "A new distance measure of belief function in evidence theory," IEEE Access, vol. 7, pp. 68 607-68 617, 2019.

[49] W. Liu, "Analyzing the degree of conflict among belief functions," Artificial Intelligence, vol. 170, no. 11, pp. 909-924, 2006.

[50] M. Daniel, "Conflicts within and between belief functions," in International Conference on Information Processing and Management of Uncertainty in KnowledgeBased Systems. Springer, 2010, pp. 696-705.

[51] E. Lefèvre and Z. Elouedi, "How to preserve the conflict as an alarm in the combination of belief functions?" Decision Support Systems, vol. 56, pp. 326-333, 2013.

[52] M. Ma and J. An, "Combination of evidence with different weighting factors: a novel probabilistic-based dissimilarity measure approach," Journal of Sensors, vol. 2015, 2015.

[53] F. Xiao, "A new divergence measure for belief functions in D-S evidence theory for multisensor data fusion," Information Sciences, vol. 514, pp. 462-483, 2020.

[54] Y. Song and Y. Deng, "Divergence measure of belief function and its application in data fusion," IEEE Access, vol. 7, no. 1, pp. $107465-107472,2019$.

[55] Y. Song, X. Wang, L. Lei, and A. Xue, "Evidence combination based on credibility and separability," in 2014 12th International Conference on Signal Processing (ICSP). IEEE, 2014, pp. 1392-1396.

[56] L. Pan and Y. Deng, "An association coefficient of belief function and its application in target recognition system," International Journal of Intelligent Systems, vol. 35, pp. 85-104, 2020.

[57] J. Zhu, X. Wang, and Y. Song, "Evaluating the reliability coefficient of a sensor based on the training data within the framework of evidence theory," IEEE Access, vol. 6, pp. 30 592-30601, 2018.

[58] D. Li and Y. Deng, "A new correlation coefficient based on generalized information quality," IEEE Access, vol. 7, no. 1, pp. 175 411-175419, 2019.

[59] B. Kang and Y. Deng, "The maximum Deng entropy," IEEE Access, vol. 7, no. 1, pp. 120758-120 765, 2019.

[60] W. Jiang, C. Huang, and X. Deng, "A new probability transformation method based on a correlation coefficient of belief functions," International Journal of Intelligent Systems, vol. 34, pp. 1337-1347, 2019.

[61] L. Fan and Y. Deng, "Determine the number of unknown targets in Open World based on Elbow method," IEEE Transactions on Fuzzy Systems, p. DOI: 10.1109/TFUZZ.2020.2966182, 2020.

[62] X. Wang and Y. Song, "Uncertainty measure in evidence theory with its applications," Applied Intelligence, vol. 48, no. 7, pp. 1672-1688, 2018.

[63] Z. Cao, W. Ding, Y.-K. Wang, F. K. Hussain, A. Al-Jumaily, and C.-T. Lin, "Effects of Repetitive SSVEPs on EEG Complexity using Multiscale Inherent Fuzzy Entropy," Neurocomputing, p. DOI: 10.1016/j.neucom.2018.08.091, 2019.

[64] C. Fu, W. Chang, M. Xue, and S. Yang, "Multiple criteria group decision making with belief distributions and distributed preference relations," European Journal of Operational Research, vol. 273, no. 2, pp. 623-633, 2019.

[65] Z. Liu, X. Zhang, J. Niu, and J. Dezert, "Combination of classifiers with different frames of discernment based on belief functions," IEEE Transactions on Fuzzy Systems, p. DOI: 10.1109/TFUZZ.2020.2985332, 2020.

[66] Z.-G. Liu, G. Qiu, G. Mercier, and Q. Pan, "A transfer classification method for heterogeneous data based on evidence theory," IEEE Transactions on Systems, Man, and Cybernetics: Systems, p. DOI: 10.1109/TSMC.2019.2945808, 2019.

[67] M. Zhou, X. Liu, and J. Yang, "Evidential reasoning approach for MADM based on incomplete interval value," Journal of Intelligent \& Fuzzy Systems, vol. 33, no. 6, pp. 3707-3721, 2017.

[68] C. Fu, M. Xue, D.-L. Xu, and S.-L. Yang, "Selecting strategic partner for tax information systems based on weight learning with belief structures," International Journal of Approximate Reasoning, vol. 105, pp. 66-84, 2019.

[69] X. Xu, H. Xu, C. Wen, J. Li, P. Hou, and J. Zhang, “A 
belief rule-based evidence updating method for industrial alarm system design," Control Engineering Practice, vol. 81, pp. 73-84, 2018.

[70] X. Xu, S. Li, X. Song, C. Wen, and D. Xu, "The optimal design of industrial alarm systems based on evidence theory," Control Engineering Practice, vol. 46, pp. 142156, 2016.

[71] Z. Luo and Y. Deng, "A matrix method of basic belief assignment's negation in Dempster-Shafer theory," IEEE Transactions on Fuzzy Systems, vol. 27, p. DOI: 10.1109/TFUZZ.2019.2930027, 2019.

[72] X. Deng and W. Jiang, "On the negation of a Dempster-Shafer belief structure based on maximum uncertainty allocation," Information Sciences, p. DOI: 10.1016/j.ins.2019.12.080, 2020.

[73] X. Gao, F. Liu, L. Pan, Y. Deng, and S.-B. Tsai, "Uncertainty measure based on Tsallis entropy in evidence theory," International Journal of Intelligent Systems, vol. 34, no. 11, pp. 3105-3120, 2019.

[74] H. Seiti, A. Hafezalkotob, S. Najafi, and M. Khalaj, "A risk-based fuzzy evidential framework for FMEA analysis under uncertainty: An interval-valued DS approach," Journal of Intelligent \& Fuzzy Systems, vol. 35, no. 2, pp. 1419-1430, 2018.

[75] Y. Song, X. Wang, L. Lei, and S. Yue, "Uncertainty measure for interval-valued belief structures," Measurement, vol. 80, pp. 241-250, 2016.

[76] M. Zhou, X.-B. Liu, Y.-W. Chen, X.-F. Qian, J.-B. Yang, and J. Wu, "Assignment of attribute weights with belief distributions for MADM under uncertainties," Knowledge-Based Systems, vol. 189, p. 105110, 2020.

[77] L. Fei and Y. Deng, "Multi-criteria decision making in Pythagorean fuzzy environment," Applied Intelligence, vol. 50, no. 2, pp. 537-561, 2020.

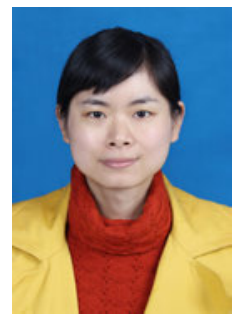

Fuyuan Xiao (M'19) received a D.E. degree in computer science and communication engineering from the Graduate School of Science and Technology, Kumamoto University, Kumamoto, Japan, in 2014. Since 2014, she has been with the School of Computer and Information Science, Southwest University, Chongqing, China, where she is an Associate Professor. She has published over 40 papers in prestigious journals and conferences, including Information Fusion, IEEE Transactions on Neural Networks and Learning Systems, IEEE Transactions on Fuzzy Systems, IEEE Transactions on Systems, Man, and Cybernetics: Systems, Information Sciences, Applied Soft Computing, Engineering Applications of Artificial Intelligence, IEICE Transactions on Information and Systems, and Artificial Intelligence in Medicine. Her current research interests include information fusion, intelligent information processing, complex event processing, and quantum decisions. Dr. Xiao severs as a Reviewer for prestigious journals, such as IEEE Transactions on Fuzzy Systems, IEEE Transactions on Cybernetics, IEEE Transactions on Pattern Analysis and Machine Intelligence, IEEE Transactions on Neural Networks and Learning Systems, Information Sciences, Knowledge-Based Systems, and Engineering Applications of Artificial Intelligence.

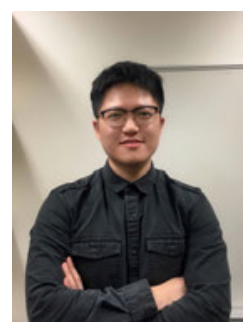

Zehong Cao (M'17) is a Lecturer (a.k.a. Assistant Professor) with the Discipline of Information and Communication Technology (ICT), School of Technology, Environments and Design, College of Sciences and Engineering, University of Tasmania (UTAS), Hobart, Australia, and an Adjust Fellow with School of Computer Science, Faculty of Engineering and Information Technology, University of Technology Sydney (UTS), Australia. He received a Ph.D. degree in information technology from UTS, and received M.S. and B.S. degrees in electronic engineering from The Chinese University of Hong Kong and Northeastern University (China), respectively. He serves as the Associate Editor of Nature Scientific Data (2019-), Journal of Journal of Intelligent and Fuzzy Systems (2019-) and IEEE Access (2018-2019), and the Guest Editor of IEEE Transactions on Emerging Topics in Computational Intelligence (2019), Swarm and Evolutionary Computation (2019), and Neurocomputing (2018). He has 40+ papers published in well-known conferences, such as AAMAS, IJCNN, IEEEFUZZY, and top-tier journals, such as IEEE TFS, TNNLS, TCYB, TSMC-S, TBME, TCDS, TITS, TII, TIA, IoT, IEEE/ACM TCBB, ACM TOMM, TOIT, Elsevier INS, NC, IJNS, NeuroImage and Nature: Scientific Data, of which 2 are ESI highly cited papers (2019-2020). He was awarded the UTS Centre for Artificial Intelligence Best Paper Award, UTS Faculty of Engineering and I.T. Publication Award, and UTS President Scholarship. His research interests cover the brain-computer interface, computational intelligence, and machine learning. He is currently focusing on the capacity of "Human-In-The-Loop" machine learning and applications.

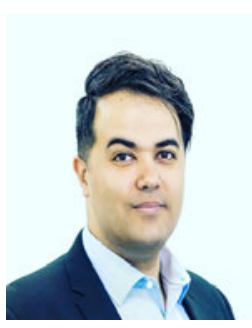

Alireza Jolfaei (SM'19) received a Ph.D. degree in applied cryptography from Griffith University, Gold Coast, Australia. He is a Lecturer (Assistant Professor in North America) and a Program Leader of Cyber Security at Macquarie University, Sydney, Australia. Before this appointment, he worked as an Assistant Professor at Federation University Australia and Temple University in Philadelphia, USA. His current research areas include cyber security, IoT security, human-in-the-loop CPS security, cryptography, A.I., and machine learning for cyber security. He has authored over 60 peer-reviewed articles on topics related to cybersecurity. He has received multiple awards for Academic Excellence, University Contribution, and Inclusion and Diversity Support. He received the prestigious IEEE Australian council award for his research paper published in the IEEE Transactions on Information Forensics and Security. He received a recognition diploma with a cash award from the IEEE Industrial Electronics Society for his publication at the 2019 IEEE IES International Conference on Industrial Technology. He is a founding member of the Federation University IEEE Student Branch. He served as the Chairman of the Computational Intelligence Society in the IEEE Victoria Section and also as the Chairman of Professional and Career Activities for the IEEE Queensland Section. He has served as the guest associate editor of IEEE journals and transactions, including the IEEE IoT Journal, IEEE Sensors Journal, IEEE Transactions on Industrial Informatics, IEEE Transactions on Industry Applications, IEEE Transactions on Intelligent Transportation Systems, and IEEE Transactions on Emerging Topics in Computational Intelligence. He has served at over 10 conferences in leadership capacities, including program co-Chair, track Chair, session Chair, and Technical Program Committee member, including IEEE TrustCom and IEEE INFOCOM. He is a Senior Member of the IEEE and an ACM Distinguished Speaker on the topic of cyber-physical systems security. 